AUTARQUIA ASSOCIADA À UNIVERSIDADE DE SÃO PAULO

DESENVOLVIMENTO DE UM REATÍMETRO DIGITAL SUBCRÍTICO BASEADO NO MODELO DE CINÉTICA PONTUAL DE GANDINI

Letícia Negrão Pinto

Tese apresentada como parte dos requisitos para obtenção do Grau de Doutor em Ciências na Área de Tecnologia Nuclear - Reatores

Orientador:

Prof. Dr. Adimir dos Santos 
INSTITUTO DE PESQUISAS ENERGÉTICAS E NUCLEARES

Autarquia associada à Universidade de São Paulo

\section{DESENVOLVIMENTO DE UM REATÍMETRO DIGITAL SUBCRÍTICO BASEADO NO MODELO DE CINÉTICA PONTUAL DE GANDINI}

Letícia Negrão Pinto

Tese apresentada como parte dos requisitos para obtenção do Grau de Doutor em Ciências na Área de Tecnologia Nuclear - Reatores

Orientador:

Prof. Dr. Adimir dos Santos

Versão Corrigida

Versão Original disponível no IPEN

São Paulo

2017 
Ao meu pai, in memoriam. 


\section{Agradecimentos}

Agradeço, primeiramente, à minha mãe, por ter sempre me apoiado.

Ao Dr. Eduardo Gonnelli, pelo apoio, companheirismo e pelas discussões produtivas

que resultaram em parceria científica, artigos, trabalhos, e muitas apresentações em congressos pelo mundo.

Ao meu orientador Dr. Adimir dos Santos e à equipe do reator nuclear IPEN/MB-01 pelo profissionalismo e colaboração.

Agradeço, finalmente, à CAPES/ELETROBRAS ELETRONUCLEAR e ao IPEN/CNEN-USP pela bolsa de estudos e apoio financeiro recebidos. 


\title{
DESENVOLVIMENTO DE UM REATÍMETRO DIGITAL SUBCRÍTICO BASEADO NO MODELO DE CINÉTICA PONTUAL DE GANDINI
}

\section{LETÍCIA NEGRÃO PINTO}

\begin{abstract}
RESUMO
Sistemas multiplicativos subcríticos foram, por muito tempo, pouco estudados, de modo que sua descrição teórica permanece com muitas questões em aberto. A presente Tese apresenta o um reatímetro subcrítico, um software desenvolvido em LabVIEW $^{\mathrm{TM}}$ capaz de medir as mudanças no fluxo de nêutrons em regime subcrítico e de transformar este sinal em reatividade. A principal vantagem de um dispositivo como este é aumento da precisão das medidas de reatividade. É sabido que reatímetros convencionais não são capazes de operar em regime subcrítico nem de descrever a dinâmica de sistemas multiplicativos nessas condições, pela própria estrutura das equações de cinética pontual clássicas em que são baseados. Diversas teorias têm sido propostas para descrever a física de sistemas subcríticos, no entanto, a maior parte envolve parâmetros extremamente complexos para serem aplicados em situações práticas. A teoria de Gandini difere das outras por ser baseada em parâmetros simples, o que a torna a escolha mais conveniente para o desenvolvimento de um reatímetro subcrítico e suas aplicações. Desta maneira, o reatímetro subcrítico desenvolvido é baseado na teoria de cinética de sistemas subcríticos de Gandini e em resultados experimentais, de altíssima qualidade e relevância, alcançados no próprio IPEN/MB-01. Deve-se destacar que a aplicação de equações de cinética específicas para sistemas subcríticos é um fato inédito no desenvolvimento de reatímetros e apenas desta maneira a reatividade pode ser estimada com precisão neste regime. O presente estudo é especialmente focado na aplicação em reatores de fissão, o qual poderá se utilizado em inúmeros experimentos em reatores de pesquisa. Portanto, o presente trabalho tem relevância tanto teórica quanto prática, já que discute um ponto fundamental da física de reatores nucleares.
\end{abstract}




\title{
DEVELOPMENT OF A DIGITAL SUBCRITICAL REACTIVITY METER BASED ON THE GANDINI'S SUBCRITICAL KINETIC THEORY
}

\section{LETÍCIA NEGRÃO PINTO}

\begin{abstract}
Multiplying subcritical systems were for a long time, poorly studied, and its theoretical description remains with plenty open questions. This Thesis presents a subcritical reactivity meter, a software developed in LabVIEW ${ }^{\mathrm{TM}}$ capable of measuring changes in neutron flux in subcritical regime and to transform this signal into reactivity. The main advantage of such device is the increase in accuracy of reactivity measurements. It is known that conventional reactivity meters cannot operate in subcritical regime nor describe the dynamics of multiplying systems under these conditions by the very structure of classical point kinetics equations. Many theories have been proposed to describe subcritical systems but most involve very complex parameters to be applied in practical situations. Gandini's theory differs from others by being based on simple parameters, being considered the most convenient choice to be made for the development of a reactivity meter and its applications. The developed subcritical reactivity meter was based on the Gandini's subcritical kinetic theory and on experimental results, of high quality and relevance, achieved at the IPEN/MB-01 reactor. It should be highlighted that the utilization of specific kinetic equations for subcritical systems is an unprecedented event in the development of reactivity meters and only by this manner the reactivity can be precisely estimated in this regime. The present study is mainly addressed to fission reactors, and it could be employed in numerous experiments in research reactors. Therefore, this work has a theoretical and practical relevance, since it discusses a fundamental point of the nuclear reactor physics.
\end{abstract}




\section{SUMÁRIO}

1. Introdução........................................................................................................................ 3

1.1. O Meio Multiplicativo Subcrítico......................................................................... 6

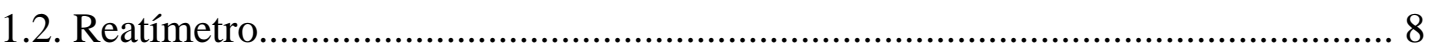

1.3. Reatímetro Subcrítico................................................................................ 9

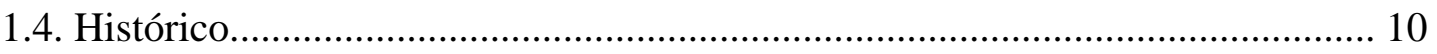

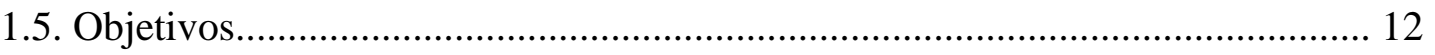

1.6. Justificativa e Originalidade....................................................................... 12

2. Reatímetro..................................................................................................................................... 13

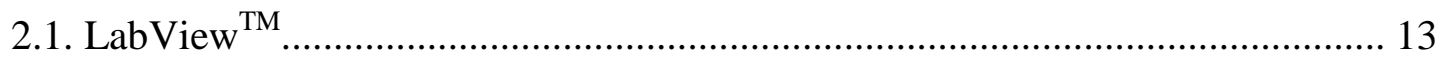

2.2. Equações do Reatímetro................................................................................ 15

2.3. Obtenção do índice de Subcriticalidade............................................................. 20

3. Fontes de Nêutrons....................................................................................................................... 23

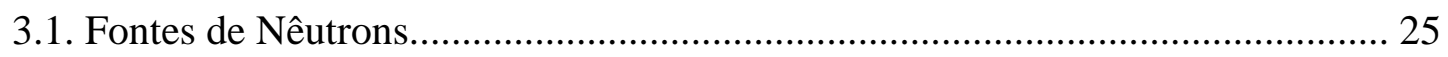

3.2. Fontes de Nêutrons Intrínsecas....................................................................... 26

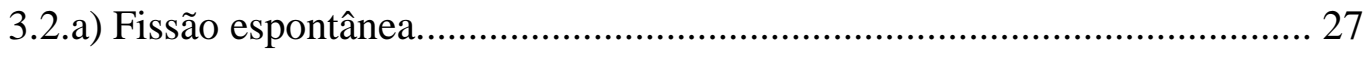

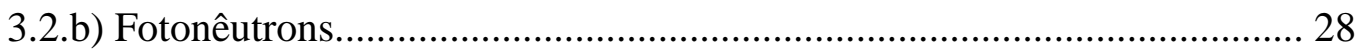

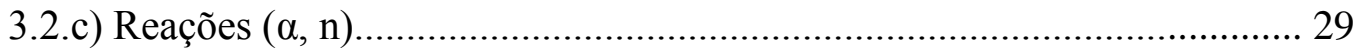

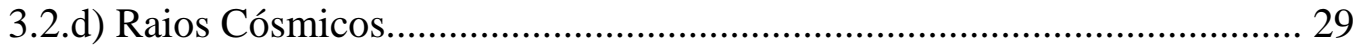


3.3. Importância da Fonte para o cálculo da Reatividade no Regime Subcrítico...... 30

3.4. Determinação da Intensidade da Fonte de Nêutrons.......................................... 31

3.5. Resumo das Etapas de Cálculo do Reatímetro.............................................. 34

4. Experimentos no Reator INPEN/MB-01......................................................... 36

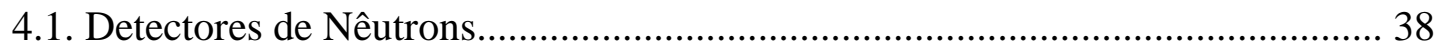

4.2. Parâmetros cinéticos do reator IPEN/MB-01................................................. 39

4.3. Experimento de Queda de Barras (Rod Drop)

4.4. Experimento de Criticalização.......................................................................... 41

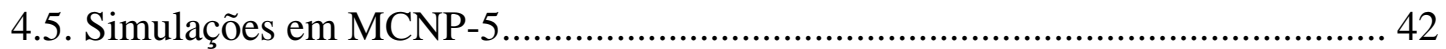

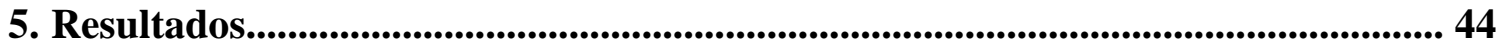

5.1. Reatímetro - Teste de Consistência.............................................................. 44

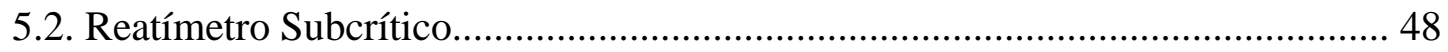

5.3. Determinação da intensidade da fonte intrínseca de nêutrons.......................... 49

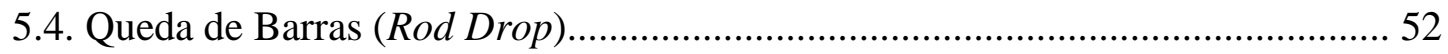

5.5. Determinação da reatividade generalizada.................................................. 56

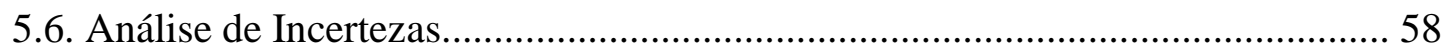

6. Discussão e Conclusão.............................................................................................. 60

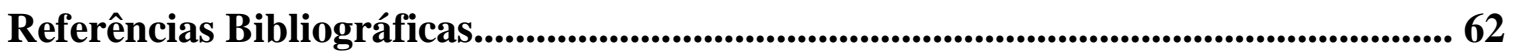




\section{Capítulo 1}

\section{Introdução}

Nos últimos anos, tem havido um grande interesse em sistemas subcríticos ${ }^{\mathrm{i}}$. Em um sistema multiplicativo subcrítico, uma reação de fissão nuclear em cadeia não consegue manter-se de maneira auto-sustentada ${ }^{\mathrm{ii}}$. No entanto, isto não quer dizer que o sistema subcrítico não seja multiplicativo. Ao se injetar no sistema subcrítico nêutrons, estes vão se multiplicar por reações de fissão em cadeia, e considerando os materiais envolvidos em um núcleo de um reator nuclear, fissões espontâneas podem sustentar certa população de nêutrons (extremamente baixa), que será mantida de modo permanente, dando origem ao que se entende por fonte intrínseca de nêutrons. Desta maneira, pode-se dizer que as propriedades de um meio multiplicativo subcrítico exigem, para se revelar, a presença de uma fonte.

Um interesse maior por sistemas subcríticos surgiu parcialmente devido ao surgimento de concepções híbridas denominadas de Accelerator-Driven Systems (ADS) ${ }^{\mathrm{iii}}$, onde há o 
acoplamento entre um reator de fissão e um acelerador de partículas. Estes sistemas híbridos propõem uma gama de vantagens importantes sobre reatores convencionais. Isto inclui uma maior segurança (e, portanto uma maior aceitação pública da energia nuclear), menores problemas com relação à proliferação, redução significativa da produção de rejeitos nucleares e ainda a possibilidade de utilizá-los para a transmutação do rejeito nuclear já existente. A física dos ADS e de seu núcleo subcrítico é bem conhecida, e existem várias publicações que lidam extensivamente com o assunto. No entanto, muitos conceitos são novos e seu entendimento requer validação experimental.

Há a necessidade do estudo e de uma maior compreensão de núcleos subcríticos também em outras situações, como é o caso da criticalização de um PWR em seus testes físicos de partida. Nesses testes, são realizados cálculos para assegurar que o núcleo pode ser operado com segurança até ser desligado para uma nova troca de combustível. No entanto, antes de ser operado a toda potência, o núcleo deve ser testado (devem-se medir certas características neutrônicas) para garantir que o novo núcleo corresponda às previsões. Deste modo, se as medidas concordarem com as predições com uma tolerância predeterminada, então há segurança para a operação do reator. Nessas situações, a reatividade é monitorada pelo método da multiplicação inversa ${ }^{\mathrm{iv}}$. Contudo, a determinação da reatividade é efetuada apenas de maneira relativa, pois não existem métodos para determinar a reatividade inicial do processo de criticalização.

Um dos fatores que contribui para que a verificação e validação dos modelos propostos para tratar de sistemas subcríticos sejam difíceis e complexas é a ausência de experimentos 
adequados que forneçam grandezas físicas que sejam descritas e possíveis de serem tratadas por esses modelos. Nesse aspecto o reator nuclear IPEN/MB-01 pode ser de grande utilidade e extrema importância por se tratar de uma instalação com características geométricas e materiais muito bem estabelecidas. O reator IPEN/MB-01 tem sido considerado benchmark internacional em vários experimentos de configurações críticas bem como em vários outros experimentos clássicos de Física de reatores ${ }^{\vee}$. Desta forma, o reator IPEN/MB-01 pode ser considerado crucial para se estabelecer parâmetros experimentais para a validação de modelos de cinéticas de sistemas subcríticos.

A reatividade subcrítica está intimamente relacionada com o modelo de cinética aplicável ao sistema. Assim, as equações de cinética pontual são fundamentais para continuamente monitorar o comportamento da reatividade para uma possível variação da intensidade das fontes externas. Neste caso, predizer os transientes de potência e reatividade de forma rápida e acurada torna-se extremamente necessário.

Considerando-se este contexto, vários modelos, como o de Gandini, por exemplo, foram propostos para caracterizar a cinética de sistemas multiplicativos subcríticos, principalmente no que tange ao item reatividade do sistema. Neste caso, os modelos teóricos sugerem o desdobramento da reatividade do sistema em duas componentes: a primeira é a reatividade do sistema conforme obtida normalmente por meio da teoria da perturbação generalizada; a segunda componente é a reatividade devido à presença da fonte no sistema. Deve-se ressaltar, ainda, que esta última componente é considerada de extrema 
complexidade para obtenção experimental devido à questão da eficiência do detector, que é alterada quando o grau de subcriticalidade do sistema se altera.

O modelo Gandini foi escolhido especificamente devido ao fato de que considera as principais características do sistema multiplicativo subcrítico. Um detalhe altamente importante é que parâmetros deste modelo são definidos exclusivamente com respeito a uma dada resposta do sistema ou a uma quantidade mensurável, como taxas de contagem dos detectores.

\subsection{O Meio Multiplicativo Subcrítico}

O estudo dos fenômenos que ocorrem em um meio multiplicativo subcrítico é útil de diversas maneiras, sobretudo quando se procura levar o sistema ao estado crítico e particularmente quando esta operação é realizada pela primeira vez. Neste caso, o problema prático consiste em prever a criticalidade do sistema e agir tempestivamente, pois, por segurança, não se pode permitir que se passe muito da previsão. Adicionalmente, é sabido que a determinação experimental da criticalidade é efetuada passando-se por estados subcríticos, sendo, portanto, de grande vantagem conhecer ao máximo as propriedades do meio multiplicativo subcrítico.

Como é conhecido, uma reação de fissão em cadeia não pode se manter em um meio subcrítico de maneira auto-sustentada. No entanto, isto não quer dizer que o sistema 
subcrítico não seja multiplicativo, isto significa apenas que seu coeficiente de multiplicação efetivo, $\mathrm{k}_{\text {eff }}$, é inferior à unidade.

Ao injetar-se em um meio multiplicativo subcrítico um feixe de nêutrons, estes vão se multiplicar por reações de fissão em cadeia. Pensando apenas neste aspecto do problema, tende-se a crer que as fissões se extinguirão após certo tempo e que os nêutrons presentes no meio multiplicativo eventualmente desaparecerão. No entanto, não é isto que acontece: nêutrons desaparecerão mas também surgirão no sistema. Nota-se que sempre existirá certa quantidade de nêutrons, mesmo quando o reator nuclear estiver completamente desligado. Este fato deve-se à própria natureza dos materiais envolvidos e estes nêutrons são, portanto, considerados uma fonte intrínseca.

Desta maneira, independentemente do que possa ser feito, o meio multiplicativo será sempre alimentado continuamente por nêutrons que não necessariamente são originários de uma fissão provocada por outro nêutron. Assim, mesmo um sistema em um estado profundamente subcrítico, os nêutrons produzidos pela fonte sustentarão a população de nêutrons e haverá um equilíbrio. ${ }^{\text {vi }}$ 


\subsection{Reatímetro}

Durante a operação de um reator nuclear de potência ou em experimentos, sinais indicando a potência e o período do reator são geralmente utilizados para a obtenção de informações diretas sobre seu estado. No entanto, o parâmetro dependente do tempo considerado mais importante é a reatividade do sistema e, consequentemente, a obtenção contínua de informações sobre o seu valor é extremamente útil e desejável.

Nesse sentido, com o intuito de medir a reatividade do sistema, de instante em instante durante a operação de um reator nuclear, o cálculo em tempo real baseado em equações cinéticas deve ser realizado. Para tanto, utiliza-se um Reatímetro.

Um Reatímetro, ou medidor de Reatividade, é um dispositivo capaz de medir as mudanças no fluxo de nêutrons. Pode ser utilizado no auxílio de operadores de centrais nucleares em abordagens subcríticas e em casos de divergências ao avaliar as respostas do reator nuclear devidas às oscilações da temperatura, ao movimento de barras de controle, ou mesmo à adição de boro no moderador. Reatímetros são comumente utilizados no processo de inicialização do reator nuclear após o reabastecimento de material combustível ou durante qualquer tipo de interrupção. Já em instalações de pesquisa, um Reatímetro pode ser utilizado em muito mais ocasiões, como para calibração de barras de controle, medida do coeficiente de reatividade, entre outras. 
Há uma demanda recente e crescente para a redução do período em que os testes, ou processos de inicialização sejam realizados. A tendência, por motivos econômicos, é de que estes períodos sejam os menores possíveis, sempre mantendo a acurácia nas medidas requeridas e adquirindo informações vitais de características neutrônicas com cada vez mais detalhe e eficiência ${ }^{\mathrm{vii}}$.

Poderia-se pensar em avaliar a reatividade de um reator através de seu período. Este pode sim ser considerado uma medida bruta da reatividade, desde que o reator esteja próximo à criticalidade e os fenômenos transientes devido aos nêutrons atrasados tenham já decaído. No entanto, o sinal de período é muito diferente daquele da reatividade em estados transientes ou subcríticos. Deste modo, os chamados "Reatímetros Subcríticos" têm sido estudados com o objetivo de eliminar esta ambiguidade para a medida precisa da reatividade nesses estados ${ }^{\text {viii }}$.

\subsection{Reatímetro Subcrítico}

Por uma questão de segurança de criticalidade, é extremamente importante monitorar a subcriticalidade adequadamente, muitas vezes com o objetivo de deixá-la sempre abaixo de um limite determinado para evitar acidentes. Em 1999, em Tokai - Mura, no Japão, um acidente de criticalidade sucedeu operações de dissolução de pó de urânio enriquecido em ácido nítrico ${ }^{\mathrm{ix}}$. Esse acontecimento demonstrou que um dispositivo de monitoramento de reatividade no regime subcrítico deve ser essencial em instalações nucleares. Nesse contexto, deve-se monitorar a subcriticalidade durante períodos de 
inspeção e troca de combustível, fabricação de combustível, plantas de reprocessamento e piscinas para a deposição de combustível queimado ${ }^{\mathrm{x}}$.

Pode-se dizer, resumidamente, que Reatímetros convencionais não conseguem operar no regime da subcriticalidade pela própria estrutura das equações de cinética clássica, que simplesmente não descrevem a dinâmica dos sistemas multiplicativos nessas condições $^{\mathrm{xi}}$.

\subsection{Histórico}

Desde os primórdios da era nuclear, a Reatividade é considerada um dos parâmetros mais importantes de um reator e, assim, a utilização de medidores de reatividade teve seu início no começo dos anos 1950. Naquele tempo, não havia um método ou dispositivo capaz de medir com precisão o parâmetro, de tal forma que a reatividade era medida

através do método do tempo de dobramento, por exemplo ${ }^{\text {xii,xiii }}$. Em seguida, a medida da reatividade evoluiu para o emprego de um computador para derivar instantaneamente a reatividade a partir do comportamento temporal da potência do reator ${ }^{\mathrm{xiv}}$.

$\mathrm{Na}$ atualidade, reatímetros subcríticos são tema de pesquisas intensas em todo o mundo. Sob a perspectiva teórica, da descrição física dos sistemas multiplicativos subcríticos, podem-se destacar diversas teses de doutorado da COPPE - Instituto Alberto Luiz Coimbra de Pós-Graduação e Pesquisa de Engenharia da Universidade Federal do Rio 
de Janeiro - UFRJ ${ }^{\mathrm{xv}, \mathrm{xvi}}$, e diversas publicações internacionais, em especial os modelos propostos por Dulla, Ravetto e Nishihara ${ }^{\text {xvii,xviii }}$.

Já sob o ponto de vista experimental, em que o desenvolvimento de um reatímetro subcrítico está sendo almejado, podem-se citar trabalhos como "An analysis of reactivity prediction during the reactor start-up process.", de BAJGL et al, de 2015 ${ }^{\mathrm{xix}}$. Neste aspecto, a publicação "Subcritical Reactivity Measurements at ANGRA I Nuclear Power Plant", de Kuramoto e Miranda, é especialmente interessante, pois comprova a necessidade de um reatímetro subcrítico na usina de ANGRA I, no Brasil ${ }^{\mathrm{xx}}$.

Com relação à aplicação industrial, empresas como a Rolls-Royce, inclusive, vendem seus reatímetros para instalações nucleares em todo o mundo, incluindo: França, China, República Tcheca, Bulgária, África do Sul, Coreia do Sul, Armênia, Espanha e Taiwan ${ }^{\text {xxi }}$. Esses reatímetros comercializados são utilizados como função permanente de segurança em reatores rápidos e, em reatores do tipo PWR, a reatividade é medida para validação do núcleo após a troca de combustível. 


\subsection{Objetivos}

Os objetivos desta tese são a elaboração de um programa de computador em linguagem LABVIEW ${ }^{\mathrm{TM}}$ para o cálculo da reatividade subcrítica baseado nas equações de cinética pontual de Gandini e o estudo e a determinação da intensidade da fonte intrínseca de nêutrons do reator IPEN/MB-01.

\subsection{Justificativas e Originalidade}

A determinação da reatividade subcrítica em reatores nucleares é um procedimento que ainda se encontra totalmente em aberto. Apesar de muitos estudos estarem sendo conduzidos em todo o mundo, ainda não há, até a presente data, nem procedimento nem modelo teórico satisfatório que descreva a cinética de sistemas subcríticos. Adicionalmente, deve-se frisar que, nesta tese, divulgam-se resultados experimentais, o que é considerado muito raro em se relacionando a este tópico. Neste sentido, este trabalho visa discutir e esclarecer muitos pontos acerca desta nova física de sistemas multiplicativos subcríticos e contribuir para esta questão. 


\title{
Capítulo 2
}

\section{Reatímetro}

\author{
2.1. LabView ${ }^{\mathrm{TM}}$
}

A linguagem eleita para a elaboração do Reatímetro Subcrítico, LabVIEW ${ }^{\mathrm{TM}}$, é uma linguagem de programação gráfica que utiliza ícones em vez de linhas de texto para criar aplicações. Assim, em contraste com as linguagens de programação baseadas em texto, onde as instruções determinam a execução do programa, o LabVIEW ${ }^{\mathrm{TM}}$ usa o fluxo de dados de programação, no qual o que determina a execução é o fluxo de dados.

Deve se destacar que, em todo o mundo, pesquisas têm sido realizadas com grande sucesso empregando-se a linguagem LabVIEW $^{\mathrm{TM}}$ na área acadêmica ${ }^{\mathrm{xxii}}$. Uma das principais vantagens em sua aplicação é que esta mesma tecnologia pode ser utilizada também na fase de execução, a fase final de desenvolvimento experimental. Essa etapa final, deve-se salientar, é fortemente relacionada à indústria, além de ainda haver a 
vantagem de se poder transferir a tecnologia ao mercado mais facilmente, já que as mesmas ferramentas e plataformas são utilizadas durante todo o processo de desenvolvimento, do estudo acadêmico à aplicação industrial.

Outra vantagem muito importante que deve ser destacada é que a linguagem LabVIEW $^{\mathrm{TM}}$ possibilita uma integração fácil através da comunicação General Purpose Interface Bus (GPIB) com a instrumentação tradicional de bancada, que pode ser encontrada normalmente em laboratórios de pesquisa, facilitando muito a investigação acadêmica a ser realizada posteriormente ao desenvolvimento do reatímetro.

Desta maneira, a partir da linguagem eleita LabVIEW ${ }^{\mathrm{TM}}$, é possível construir uma interface de usuário, conhecida como o painel frontal, com um conjunto de ferramentas e objetos. O código é adicionado utilizando-se representações gráficas de funções para controlar os objetos do painel frontal. Um diagrama de blocos contém este código e um exemplo pode ser visto na Figura 2.1.-1. 


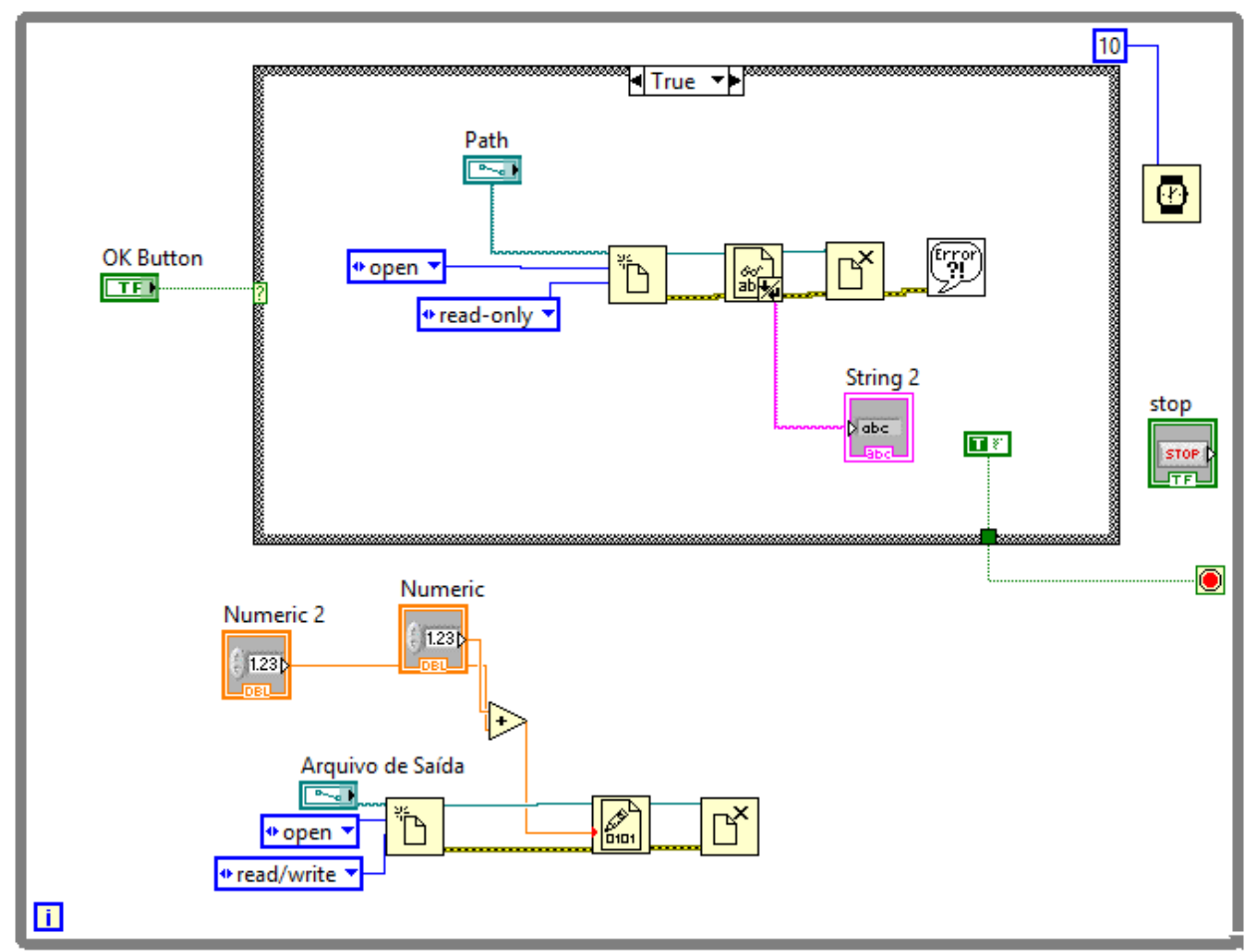

Figura 2.1.-1: Exemplo de Diagrama de Blocos do LabVIEW ${ }^{\mathrm{TM}}$.

\subsection{Equações do Reatímetro}

No desenvolvimento do algoritmo para o cálculo da reatividade subcrítica, primeiramente deve-se considerar as equações de cinética pontual para sistemas subcríticos propostas por Gandini ${ }^{\text {xiii }}$. De acordo com a cinética de sistemas subcríticos proposta por este autor, as equações para a potência relativa entre dois estados subcríticos e para a concentração dos precursores de nêutrons atrasados são dadas por:

$l_{\text {eff }} \frac{d P_{N}(t)}{d t}=\left(\rho_{\text {gen }}-\alpha \beta\right) P_{N}+\alpha \sum_{i=1}^{I} \lambda_{i} \xi_{i}+\zeta\left(1-P_{N}\right)+\rho_{\text {source }}$ 
$\frac{d \xi_{j}(t)}{d t}=\beta_{j} P_{N}(t)-\lambda_{j} \xi_{j}(t)$

Onde:

$l_{\text {eff }}$ representa o tempo de geração de nêutrons prontos;

$\boldsymbol{P}_{N}(t)$ representa a potência normalizada do reator, ou a potência relativa entre dois estados subcríticos do reator;

Deve-se ressaltar que $\boldsymbol{\rho}_{g e n}$ não é a reatividade total, mas sim a reatividade obtida após uma perturbação, ou ainda a reatividade generalizada entre dois pontos subcríticos;

$\boldsymbol{\alpha}$ representa a relação entre o espectro de nêutrons prontos e o espectro de nêutrons atrasados;

$\boldsymbol{\beta}$ representa a fração efetiva de nêutrons atrasados;

$\boldsymbol{\beta}_{\mathbf{j}}$ representa a fração de nêutrons atrasados da família $j$;

$\lambda_{\mathbf{j}}$ representa a constante de decaimento dos precursores de nêutrons atrasados da família $j$;

$\xi_{\mathbf{j}}$ representa a concentração dos precursores de nêutrons atrasados;

$\zeta$ representa o índice de subcriticalidade, e $\rho_{\text {source }}$ representa a reatividade devido à variação da fonte. Neste trabalho pode-se considerar que $\rho_{\text {source }}$ é nulo, pois na instalação IPEN/MB-01 não há variação da fonte. Este termo é considerado em sistemas ADS. 
Pode-se notar que, para resolver as equações (2.2.-1) e (2.2.-2), é necessário o conhecimento do índice de subcriticalidade. Conforme demonstrado por Dos Santos, A., Lee, S. M., Diniz, R. e Jerez, R., esse índice é igual à reatividade obtida da equação da cinética pontual clássica considerando a presença de uma fonte ${ }^{\mathrm{xxiv}}$.

A semelhança entre as equações subcríticas descritas anteriormente e as equações de cinética pontual clássicas é apenas aparente - elas devem ser utilizadas com cuidado já que a potência relativa e a reatividade generalizada fazem uma grande diferença entre os modelos.

Assumindo $\rho_{\text {source }}$ igual a zero, pois não haverá variação da fonte nos experimentos a serem realizados no reator IPEN/MB-01, primeiramente, resolve-se a equação diferencial número (2.2.-2):

$\xi_{j}(t)=\xi_{j}(0) e^{-\lambda_{j} t}+\beta_{j} e^{-\lambda_{j} t} \int_{t^{\prime}}^{t} P_{N}\left(t^{\prime \prime}\right) e^{\lambda_{j} t^{\prime \prime}} d t^{\prime \prime}$

Agora, explicitamos $\rho_{\text {gen }}$ da equação (2.2.-1):

$\rho_{g e n}(t)=\frac{1}{P_{N}}\left[\alpha \sum_{i=1}^{I} \lambda_{i} \xi_{i}+\zeta\left(1-P_{N}\right)-\ell_{e f f} \frac{d}{d t} P_{N}(t)\right]+\alpha \beta$ 
Substituímos a equação (2.2.-3) em (2.2.-4):

$$
\begin{aligned}
\rho_{\text {gen }}(t)=\frac{\zeta}{P_{N}}\left(1-P_{N}\right)+\alpha \beta-\frac{\ell_{e f f}}{P_{N}} \frac{d}{d t} P_{N}(t) & +\frac{\alpha}{P_{N}} \sum_{i=1}^{I} \lambda_{i} \xi_{i}(0) e^{-\lambda_{i} t} \\
& +\frac{\alpha}{P_{N}} \sum_{i=1}^{I} \lambda_{i} \beta_{i} e^{-\lambda_{i} t^{t}} \int_{t^{\prime}} P_{N}\left(t^{\prime \prime}\right) e^{\lambda i t^{\prime \prime}} d t^{\prime \prime}
\end{aligned}
$$

Supondo o reator em estado estacionário em $\mathrm{t}=0$, obtemos, para cada $\mathrm{i}$ :

$$
\xi_{i}(0)=\frac{\beta_{i}}{\lambda_{i}} P_{N}(0)
$$

Substituindo a equação (2.2.-6) em (2.2.-5), tem-se finalmente que:

$$
\begin{aligned}
\rho_{g e n}(t)=\frac{\zeta}{P_{N}}\left(1-P_{N}\right)+\alpha \beta-\frac{\ell_{e f f}}{P_{N}} & \frac{d}{d t} P_{N}(t)+\alpha \frac{P_{N}(\mathrm{O})}{P_{N}} \sum_{i=1}^{I} \beta_{i} e^{-\lambda_{i} t} \\
& +\frac{\alpha}{P_{N}} \sum_{i=1}^{I} \lambda_{i} \beta_{i} e^{-\lambda_{i} t} \int_{t^{\prime}}^{t} P_{N}\left(t^{\prime \prime}\right) e^{\lambda i t^{\prime \prime}} d t^{\prime \prime}
\end{aligned}
$$

Sendo assim, o algoritmo final para o cálculo da reatividade subcrítica é obtido a partir da equação (2.2.-7), avaliando a derivada e a integral em termos de pequenos intervalos de tempo: $t_{0}, t_{k}, t_{k+1}, \ldots, t_{k+n}$.

Definindo:

$$
A_{k}=\alpha \beta-\left.\frac{\ell_{e f f}}{P_{N_{k}}} \frac{d}{d t} P_{N}\right|_{t_{k}}
$$




$$
\begin{aligned}
& B_{k}=\frac{\zeta}{P_{N_{k}}}\left(1-P_{N_{k}}\right) \\
& D_{i, k}=\lambda_{i} \xi_{i}(0) e^{-\lambda_{i} t_{k}} \\
& F_{i, k}=\lambda_{i} \beta_{i} e^{-\lambda_{i} t_{k}} \int_{0}^{t_{k}} P_{N}\left(t^{\prime \prime}\right) e^{\lambda i t^{\prime \prime}} d t^{\prime \prime}
\end{aligned}
$$

Tem-se que a reatividade no instante $t_{k}$ pode ser dada por:

$$
\rho_{g e n_{k}}=A_{k}+B_{k}+\frac{\alpha}{P_{N_{k}}} \sum_{i=1}^{I} D_{i, k}+\frac{\alpha}{P_{N_{k}}} \sum_{i=1}^{I} F_{i, k}
$$

Já no instante imediatamente posterior, $t_{k+1}$, a reatividade pode ser dada por:

$$
\rho_{g e n_{k+1}}=A_{k+1}+B_{k+1}+\frac{\alpha}{P_{N_{k+1}}} \sum_{i=1}^{I} D_{i, k+1}+\frac{\alpha}{P_{N_{k+1}}} \sum_{i=1}^{I} F_{i, k+1}
$$

Em que:

$$
\begin{aligned}
& A_{k+1}=\alpha \beta-\left.\frac{\ell_{\text {eff }}}{P_{N_{k+1}}} \frac{d}{d t} P_{N}\right|_{t_{k+1}} \\
& B_{k+1}=\frac{\zeta}{P_{N_{k+1}}}\left(1-P_{N_{k+1}}\right) \\
& D_{i, k+1}=\lambda_{i} \xi_{i}(0) e^{-\lambda_{i} \Delta t} \\
& F_{i, k+1}=F_{i, k} e^{-\lambda_{i} \Delta t}+\lambda_{i} \beta_{i} e^{-\lambda_{i} t_{k+1}} \int_{0}^{t_{k+1}} P_{N}\left(t^{\prime \prime}\right) e^{\lambda i t^{\prime \prime}} d t^{\prime \prime}
\end{aligned}
$$


Nas expressões (2.2.-16) e (2.2.-17), tem-se que $\Delta t=\left(t_{k+1}-t_{k}\right)$ representa o intervalo de tempo utilizado pelo algoritmo do reatímetro para o cálculo da reatividade.

Assim, as equações (2.2.-13), (2.2.-14), (2.2.-15), (2.2.-16) e (2.2.-17) constituem um algoritmo tipo passo, onde a reatividade no instante $t_{k+1}$ é obtida utilizando os parâmetros conhecidos no instante imediatamente anterior $t_{k}$.

\subsection{Obtenção do índice de Subcriticalidade}

Da equação para $\rho_{\text {gen }}$ observa-se que existem duas incógnitas: a potência relativa $\mathrm{P}_{\mathrm{N}}$ e o índice de subcriticalidade $\zeta$. A potência relativa $\mathrm{P}_{\mathrm{N}}$ pode ser obtida através da razão do sinal do detector entre duas aquisições sucessivas. Como mencionado anteriormente, o índice de subcriticalidade $\zeta$ pode ser obtido por meio de um trabalho recente efetuado nas dependências do reator IPEN/MB-01: o índice corresponde à reatividade obtida pela cinética pontual clássica com fonte.

Desta maneira, um algoritmo para o cálculo do índice de subcriticalidade pode ser obtido partindo-se das equações de cinética pontual clássicas com fonte:

$\frac{d N(t)}{d t}=\frac{\rho(t)-\beta}{\Lambda} N(t)+\sum_{i=1}^{6} \lambda_{i} C_{i}(t)+S$

e 


$$
\frac{d C_{i}(t)}{d t}=\frac{\beta_{i}}{\Lambda} N(t)-\lambda_{i} C_{i}(t)
$$

Onde:

$N(t)$ é a população neutrônica no instante t;

$\rho(t)$ é a reatividade ao instante t;

$\beta$ é a fração efetiva de nêutrons atrasados.

$\beta_{i}$ é a abundância de nêutrons atrasados do i-ésimo grupo de precursores;

$\lambda_{i}$ é a constante de decaimento do i-ésimo grupo de precursores;

$C_{i}(t)$ é a concentração de precursores do i-ésimo grupo no instante t;

$\Lambda$ é o tempo de geração de nêutrons prontos;

S é a intensidade da fonte de nêutrons.

A partir de um raciocínio análogo ao usado na derivação da expressão de $\rho_{\text {gen }}$, é possível chegar à equação:

$$
\begin{aligned}
\zeta=\rho(t)=\frac{\Lambda}{N(t)} \frac{d N(t)}{d t}+\beta & -\frac{N_{0}}{N(t)} \sum_{i=1}^{I} \beta_{i} e^{-\lambda_{i} t} \\
& -\frac{1}{N(t)} \sum_{i=1}^{I} \lambda_{i} \beta_{i} e^{-\lambda_{i} t} \int_{t^{\prime}}^{t} N\left(t^{\prime \prime}\right) e^{\lambda_{i} t^{\prime \prime}} d t^{\prime \prime}-\frac{\Lambda}{N(t)} S
\end{aligned}
$$

Da teoria de física de reatores nucleares ${ }^{\mathrm{xxv}, \mathrm{xxv}}$, e do que foi desenvolvido aqui, nota-se que a reatividade do sistema pode ser obtida rearranjando-se as equações de cinética 
inversa de forma a explicitar o parâmetro em função do tempo. A partir daí, pode-se obter o algoritmo utilizado para o cálculo da reatividade subcrítica.

É importante ainda ressaltar que o algoritmo final para o cálculo da reatividade subcrítica deve considerar duas etapas de cálculos que não ocorrem simultaneamente:

> Uma primeira etapa para o cálculo do índice de subcriticalidade $\zeta$;

> E outra, que ocorre logo em seguida, utilizando-se deste índice para o cálculo da reatividade subcrítica dada pelo modelo de Gandini. 


\section{Capítulo 3}

\section{Fontes de Nêutrons}

No núcleo de um reator nuclear, ou, mais genericamente, em um meio multiplicativo, podem existir nêutrons provenientes das mais diversas origens. Os mais importantes para a maioria dos problemas da física de reatores nucleares são, obviamente, os nêutrons originados direta e indiretamente por fissões nucleares induzidas. Neste capítulo, contudo, serão tratadas justamente as outras fontes de nêutrons e sua importância para a subcriticalidade.

Reatores nucleares utilizam nêutrons que não são originários de fissões induzidas por dois motivos:

1) Para fornecer uma indicação visível para uma segura criticalização, sensibilizando os detectores de nêutrons (neste caso as chamadas Fontes Externas, ou Instaladas);

2) Para assegurar o nível mínimo necessário de nêutrons para um processo estatisticamente estável (neste caso a chamada Fonte Intrínseca). 
Neste ponto, vale lembrar que um determinado processo é considerado estável caso a análise dos dados aponte que não há presença de causas especiais de variação atuando sobre o mesmo. De outra maneira, em um processo estável os pontos nos gráficos de controle devem distribuir-se aleatoriamente em torno da linha média sem que haja padrões, tendências crescentes ou decrescentes ${ }^{x x v i i}$. Este é um conceito cuja importância será compreendida mais adiante no texto.

Nêutrons que não são provenientes de fissões induzidas são uma parte significativa e importante da população neutrônica no núcleo do reator somente durante sua criticalização e durante os últimos estágios do desligamento do reator, quando a potência decai ao menor nível de medição dos instrumentos nucleares. Obviamente, este é um nível de potência muito abaixo da potência mínima para que a criticalidade seja estabelecida e, desta maneira, este nível de potência é chamado de "Região Subcrítica". Deve-se notar que é possível para um reator estar subcrítico (estando apenas levemente abaixo da criticalidade) sem necessariamente estar na Região Subcrítica.

É justamente na Região Subcrítica que o tratamento analítico da multiplicação subcrítica da fonte de nêutrons se aplica, com a mais notável condição de equilíbrio conhecida por "equilíbrio da multiplicação subcrítica". Quando a população neutrônica atinge níveis muito baixos, os nêutrons-fonte podem compensar suas perdas pelo processo de fissão e criar uma potência de equilíbrio. 


\subsection{Fontes de Nêutrons}

Mesmo quando o reator está desligado, ou em estados profundamente subcríticos, há a presença de nêutrons provenientes das mais variadas origens, ou fontes. Alguns desses nêutrons são originados simplesmente devido à natureza dos materiais envolvidos (fonte intrínseca), enquanto outros podem ser resultado de fontes de nêutrons externas, ou instaladas, que são incorporadas ao projeto do reator ou ao experimento. Desta forma, nêutrons originados por outras fontes além da fissão induzida por nêutrons são frequentemente agrupados e classificados como nêutrons-fonte. Em outras palavras, os nêutrons-fonte podem ser sub-classificados em fontes de nêutrons intrínsecas ou externas/instaladas, como pode ser melhor compreendido através da figura 3.1.-1.

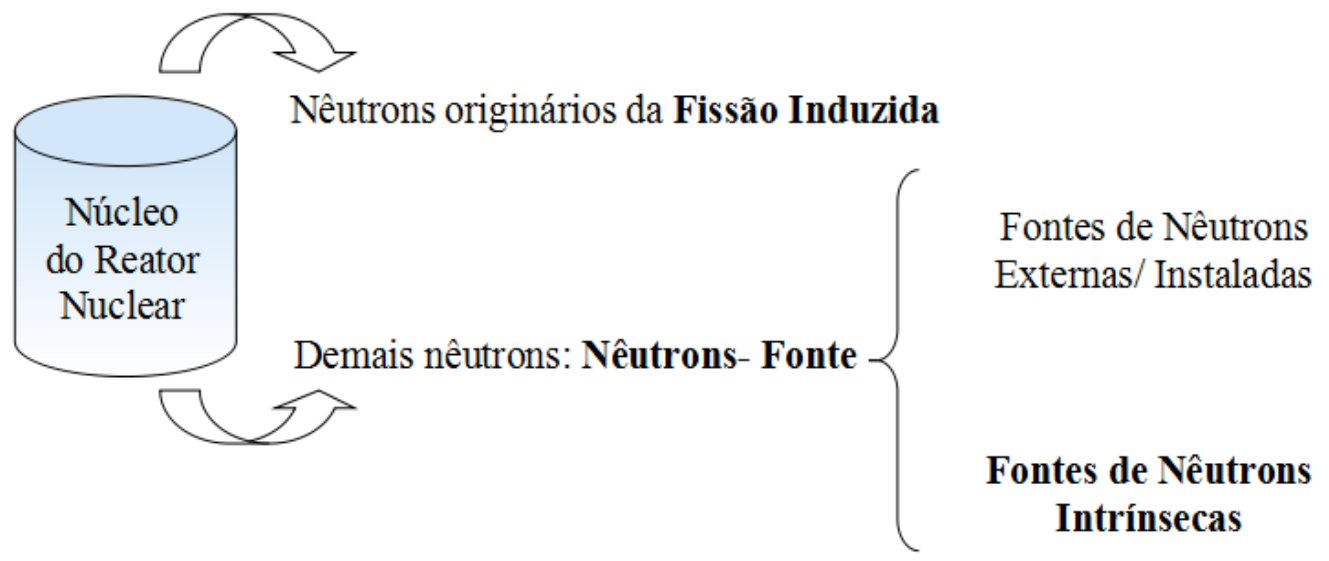

Figura 3.1.-1. Esquema representando as diversas origens dos nêutrons presentes em um reator nuclear. 
Nêutrons-fonte são importantes por garantirem que a população de nêutrons permaneça alta o suficiente de modo a permitir uma indicação visível do nível de nêutrons nos sistemas de monitoramento mais sensíveis enquanto o reator é desligado e durante sua criticalização. Isto garante a operabilidade instrumental, permite o monitoramento de mudanças na população neutrônica (fluxo de nêutrons), tendo, consequentemente, impacto na manutenção da segurança da instalação nuclear como um todo.

Com relação especificamente às fontes de nêutrons externas, artificiais, estas são consideradas, em geral, fracas, mas de intensidade relativamente constante.

\subsection{Fontes de Nêutrons Intrínsecas}

Pode ser observado que alguns nêutrons simplesmente "surgem" no reator devido a uma variedade de reações inevitáveis que ocorrem devido à própria característica dos materiais envolvidos. Como visto anteriormente, fontes de nêutrons intrínsecas são chamadas as reações que dão origem a nêutrons que sempre estarão presentes em sistemas multiplicativos.

Independente das fontes externas, ou instaladas, que podem ser introduzidas deliberadamente (Rádio-Berílio, Polônio-Berílio, ou Amerício-Berílio, no caso do reator IPEN/MB-01) sempre existirá em um meio subcrítico a presença de uma fonte intrínseca de nêutrons. 
A intensidade da fonte intrínseca pode variar dependendo da história de operação do reator, sendo significativamente mais fraca que a fonte externa, artificial, em núcleos novos de reatores nucleares. De maneira oposta, a fonte intrínseca pode ser muito mais intensa que a fonte externa no momento do desligamento do reator após uma operação à alta potência. No entanto, essa intensidade decai durante o desligamento do reator e, com o tempo, tende a ficar bem menos intensa que a fonte externa. No caso específico do reator nuclear IPEN/MB-01, como este é um reator de pesquisa de potência zero, com consumo de combustível considerado desprezível, a possível variação de intensidade da fonte intrínseca também pode ser desprezada.

Com relação aos processos físicos que dão origem aos nêutrons da fonte intrínseca, tem-se que estes são originados, principalmente, por:

\section{2.a) Fissão espontânea}

Em um arranjo com urânio natural, a fissão espontânea de ${ }^{238} \mathrm{U}$ dá origem à aproximadamente um nêutron por grama por minuto. Apesar de muito fraca, essa emissão apresenta um interesse prático, particularmente em um arranjo com grafite, onde não existem as outras possibilidades de formação de nêutrons que serão mencionados posteriormente. Desta forma, em um arranjo contendo 60 toneladas de urânio, espontaneamente, a cada segundo, são originados 1 milhão de nêutrons; o que seria suficiente para iniciar reações em cadeia e permitir o controle da aproximação às condições 
de criticalidade. Em um arranjo de ${ }^{235} \mathrm{U}$, no entanto, a emissão de nêutrons por esse processo é muito menos intensa para ser utilizada desta maneira.

Já em um arranjo de Plutônio, esta emissão depende principalmente do teor do

combustível em ${ }^{240} \mathrm{Pu}$, em que a constante de fissão espontânea é 40 mil vezes superior àquela do isótopo principal ${ }^{239} \mathrm{Pu}$.

\section{2.b) Fotonêutrons}

A energia de ligação de um nêutron é perto de $8 \mathrm{MeV}$ para a maior parte dos nuclídeos, mas de apenas 1,6 MeV no núcleo de Berílio e de 2,3 MeV naquele do Deutério. Assim, com raios gama suficientemente energéticos, tais quais os emitidos por certos produtos de fissão, pode-se, desta maneira, provocar reações de foto-fissão:

$$
\begin{gathered}
{ }^{9} \mathrm{Be}(\gamma, n){ }^{8} \mathrm{Be} \\
{ }^{2} \mathrm{D}(\gamma, \mathrm{n}) \mathrm{p}
\end{gathered}
$$

Essa emissão de fotonêutrons apenas se manifesta em um arranjo em que no moderador há a presença de Berílio ou água pesada, e deve sua importância à existência entre os produtos de fissão de emissores de gama duros de longa vida (como o ${ }^{140} \mathrm{La} \mathrm{em}$ equilíbrio com ${ }^{140} \mathrm{Ba}$ de meia vida 12,8 dias). 
Além do caso em que o arranjo é criticalizado pela primeira vez, a fonte de fotonêutrons devida aos produtos de fissão acumulados durante funcionamentos anteriores supera completamente a emissão devida à fissão espontânea.

\section{2.c) Reações $(\alpha, n)$}

Em certas condições as partículas alfa emitidas pela substância físsil podem provocar reações nucleares $(\alpha, n)$ nos núcleos presentes em sua vizinhança. Seria o caso, por exemplo, em um arranjo onde o combustível nuclear fosse utilizado em ligação com Berílio. Da mesma maneira, sugeriu-se que em meio aquoso, as reações $(\alpha, n)$ com Oxigênio dão origem a um nêutron para cada $10^{7}$ partículas alfa, aproximadamente. Assim, considerando 10 nêutrons por $\mathrm{cm}^{3}$ por segundo em uma solução de Plutônio a 50g/L, isto representaria 5 vezes a emissão devido à fissão espontânea, considerando-se $3 \%$ de ${ }^{240} \mathrm{Pu}$.

\section{2.d) Raios Cósmicos}

Os raios cósmicos são muitas vezes citados entre fontes de nêutrons possíveis. Na realidade, seu papel pode ser desprezado, pois a densidade de nêutrons de origem cósmica é muito pequena ao nível do mar e, além disso, a estrutura de concreto que protege o reator nuclear absorve completamente os nêutrons provenientes do exterior que poderiam penetrar no núcleo. 


\subsection{Importância da Fonte para o cálculo da Reatividade no Regime Subcrítico}

As equações de cinética pontual, nas quais os reatímetros convencionais são baseados, são derivadas a partir do pressuposto de que a distribuição do fluxo de nêutrons não é dependente da reatividade. No entanto, a hipótese não é verdadeira para os sistemas subcríticos (em que é considerada a presença de uma fonte de nêutrons), uma vez que a distribuição do fluxo depende da subcriticalidade do sistema ${ }^{\text {xxviii }}$.

No domínio subcrítico, ou mesmo com o reator crítico operando a uma potência muito baixa, o número total de nêutrons no núcleo é muito baixo e torna-se comparável ao número de nêutrons gerados pela presença dos nêutrons-fonte. Neste caso, pode-se afirmar que a fonte domina o comportamento do sistema multiplicativo e influencia a contagem de nêutrons e a determinação da reatividade.

Ao se trabalhar no domínio subcrítico, as condições do núcleo podem ser consideradas semelhantes às de equilíbrio (o número de nêutrons no núcleo e a concentração de precursores de nêutrons constantes no tempo). Caso o termo fonte seja ignorado, a tendência do reatímetro clássico será mostrar um resultado crescente para a reatividade do sistema, tendendo a zero, perdendo-se assim qualquer conexão com o sistema real. Desnecessário dizer que este comportamento pode levar a complicações em uma instalação nuclear. Portanto, em regime subcrítico, o termo fonte é considerado de extrema importância e jamais deve ser ignorado nas equações cinéticas. ${ }^{\text {xxix }}$. 


\subsection{Determinação da Intensidade da Fonte de Nêutrons}

A intensidade da fonte de nêutrons, denotada por S nas equações deste trabalho, não é nem deve ser confundida com a fonte de Amerício-Berílio do reator IPEN/MB-01. A intensidade da fonte de nêutrons é expressa como a taxa efetiva de nêutrons detectados devido à fonte intrínseca. Este parâmetro, que é dependente tanto das características do meio multiplicativo quanto do detector por meio da eficiência, é geralmente desconhecido. Desta forma, calcular a reatividade subcrítica significa também ter que estimar a intensidade da fonte de nêutrons intrínseca $S .^{\mathrm{xx}}$

O método eleito para a determinação da intensidade da fonte de nêutrons foi o Método dos Mínimos Quadrados em Cinética Inversa (conhecido como LSIKM) ${ }^{\mathrm{xxxi}}$. Para baixos níveis de sinal neutrônico, o grau de flutuação do sinal aumenta e causa dificuldades na avaliação da reatividade. Para o caso de uma grande mudança na reatividade, como em um experimento de queda de barras (rod drop), o Método dos Mínimos Quadrados em cinética Inversa (LSIKM) com uma função de ajuste apropriada é um modo efetivo para determinar-se a intensidade de nêutrons da fonte.

Para chegar à equação de ajuste, primeiramente deve-se considerar a equação de cinética inversa discretizada com fonte. A expressão para dados de séries temporais discretas pode ser derivada das equações (2.3.-1) e (2.3.-2), assumindo que a variação de 
potência do reator para o intervalo $\Delta \mathrm{t}$ segue $\mathrm{N}(\mathrm{t})=\mathrm{N}(\mathrm{t}-1) \quad \exp \left(\mu_{\mathrm{j}} \mathrm{t}\right)$, com $\mu_{\mathrm{j}}=\log (\mathrm{N}(\mathrm{t}) / \mathrm{N}(\mathrm{t}-1)) / \Delta \mathrm{t}:$

$\rho_{f}(t)-\beta=\frac{\Lambda}{N(t)} \cdot \frac{\Delta N(t)}{\Delta t}-\frac{\Lambda}{N(t)} \sum_{i=1}^{6} \lambda_{i} C_{i}(t)-\frac{\Lambda}{N(t)} S$

Onde:

$$
C_{i}(t)=C_{i}(t-1) e^{\left(-\lambda_{i} \Delta t\right)}+\frac{\beta_{i}}{\Lambda} \cdot \frac{N(t)-N(t-1) e^{\left(-\lambda_{i} \Delta t\right)}}{\frac{1}{N(t)} \cdot \frac{\Delta N(t)}{\Delta t}+\lambda_{i}}
$$

$\rho_{\mathrm{f}}(\mathrm{t})$ representa a reatividade dada pelas equações de cinética pontual clássicas com fonte discretizadas, e, como visto anteriormente, pode ser identificado como o índice de subcriticalidade $\zeta$.

Quando a potência do reator está em estado estacionário, as seguintes condições iniciais podem ser obtidas:

$$
C_{i, 0}=\frac{N_{0} \beta_{i}}{\lambda_{i} \Lambda} \text { e } \quad \rho_{0}=-\frac{\Lambda}{N_{0}} S
$$

Rearranjando os termos da equação (3.4.-1), tem-se que: 


$$
N(t)=\frac{\Lambda}{\rho_{f}(t)-\beta} \cdot \frac{\Delta N(t)}{\Delta t}-\frac{\Lambda}{\rho_{f}(t)-\beta} \sum_{i=1}^{6} \lambda_{i} C_{i}(t)-\frac{\Lambda}{\rho_{f}(t)-\beta} S
$$

Ou:

$$
N(t)=\frac{\Lambda}{\rho_{f}(t)-\beta}\left[\frac{\Delta N(t)}{\Delta t}-\sum_{i=1}^{6} \lambda_{i} C_{i}(t)\right]-\frac{\Lambda}{\rho_{f}(t)-\beta} S
$$

Neste ponto, introduz-se uma nova variável:

$$
Q(t)=\frac{\Delta N(t)}{\Delta t}-\sum_{i=1}^{6} \lambda_{i} C_{i}(t)
$$

Assim, substituindo a equação (3.4.-6) em (3.4.-5), chega-se finalmente a:

$$
N(t)=\frac{\Lambda}{\rho_{f}(t)-\beta} Q(t)-\frac{\Lambda}{\rho_{f}(t)-\beta} S
$$

Como a variável Q(t) é bem comportada e a distribuição de frequências de $\mathrm{N}(\mathrm{t})$ é uma distribuição normal, consequentemente a melhor combinação de variáveis é aquela em que $Q(t)$ está no eixo " $x$ ” e $N(t)$ no eixo “ $y$ "xxxii . Desta forma, quando a sequência temporal de dados de $\mathrm{Q}(\mathrm{t})$ e $\mathrm{N}(\mathrm{t})$ são plotadas nas coordenadas x e y, respectivamente, a obtenção da intensidade da fonte de nêutrons $\mathrm{S}$ pode ser realizada a partir de um ajuste de mínimos quadrados. 


\subsection{Resumo das Etapas de Cálculo do Reatímetro}

O reatímetro subcrítico desenvolvido nesta tese, como mencionado, foi programado utilizando-se a linguagem LabVIEW ${ }^{\mathrm{TM}}$ e é capaz de calcular a reatividade subcrítica de acordo com a cinética pontual de Gandini. O programa desenvolvido pode ser decomposto em três etapas principais:

$1^{\mathrm{a}}$ - Calcular um parâmetro "Q" para a determinação da intensidade da fonte intrínseca "S";

$2^{\mathrm{a}}$ - Determinar o índice de subcriticalidade " $\zeta$ " a partir das equações de cinética pontual clássicas com fonte obtida da etapa anterior;

$3^{\mathrm{a}}$ - Determinar a reatividade generalizada, $\rho_{\text {gen }}$, levando-se em conta os parâmetros previamente calculados.

Para que fiquem mais claros todos os passos a serem seguidos até a obtenção da reatividade subcrítica, o fluxograma abaixo (Figura 3.5.-1) explicita a ordem dos cálculos a serem realizados pelo reatímetro subcrítico. 


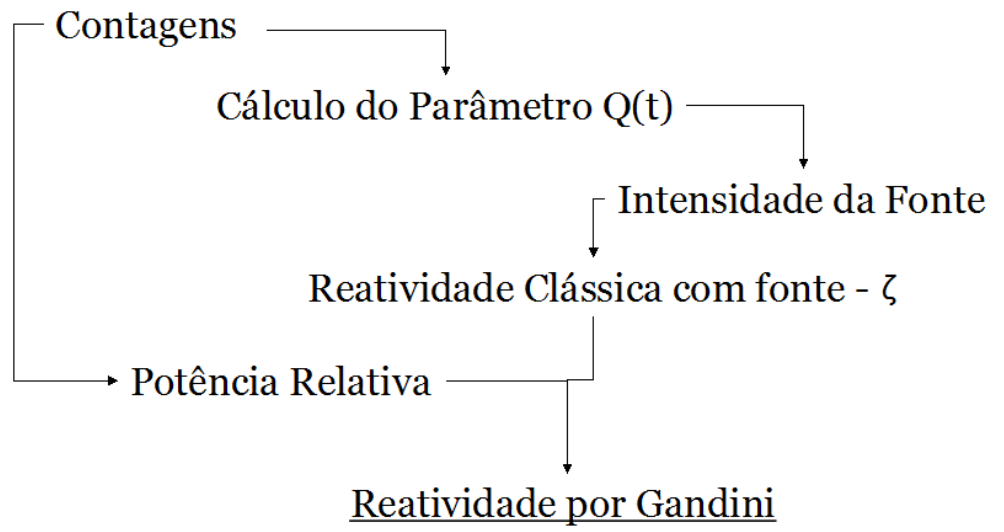

Figura 3.5.-1: Fluxograma dos processos de cálculo para a obtenção da reatividade subcrítica de acordo com a cinética pontual de Gandini a partir apenas das taxas de contagens de nêutrons. 


\section{Capítulo 4}

\section{Experimentos no Reator IPEN/MB-01}

Com o intuito de utilizar dados de entrada reais no reatímetro desenvolvido e analisar, a partir daí, os resultados para a reatividade generalizada, foram executados alguns experimentos subcríticos no reator IPEN/MB-01 do Instituto de Pesquisas Energéticas e Nucleares IPEN/CNEN-SP. Dois detectores proporcionais de nêutrons foram utilizados nos experimentos, além de serem realizadas simulações em Monte Carlo utilizando-se o código de física de reatores nucleares MCNP-5.

Todos os experimentos considerados nesta Tese foram executados no reator nuclear IPEN/MB-01 considerando-se o arranjo de núcleo padrão, de 28 x 26 varetas combustíveis e 48 tubos guia, destinados à inserção das varetas de controle e segurança, como pode ser visto na figura 4-1. 


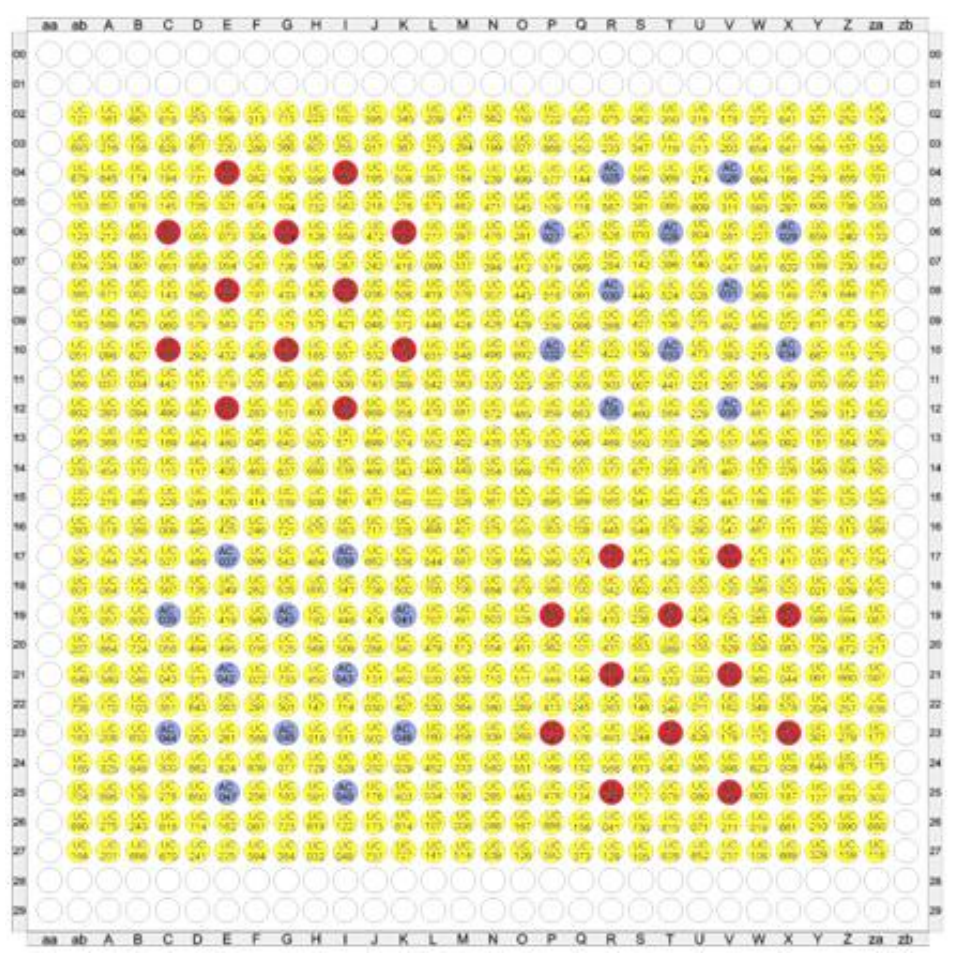

Figura 4-1: Esquema do reator IPEN/MB-01 em que se pode ver o arranjo de núcleo padrão. Em azul pode-se ver os bancos de controle e em vermelho os bancos de segurança.

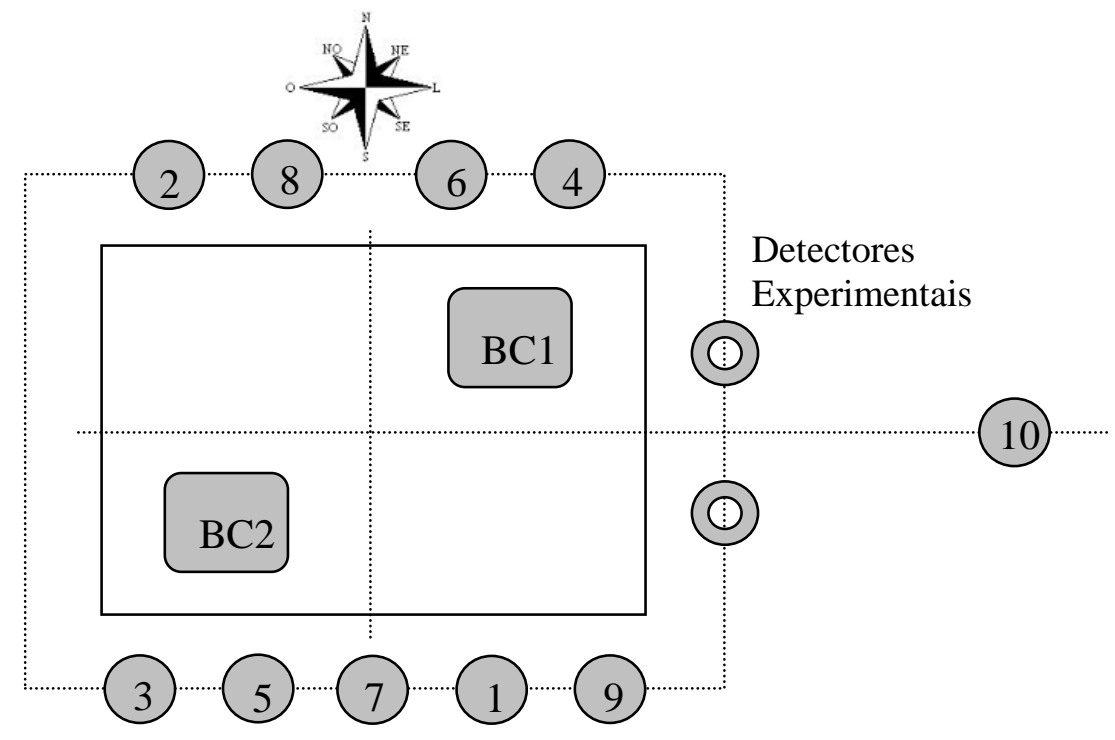

Figura 4-2: Posicionamentádeo do $\begin{array}{r}\text { Núdectores e dos bancos de controle BC1 e BC2 no } \\ \text { Reator }\end{array}$ reator IPEN/MB-01. 


\subsection{Detectores de Nêutrons}

Os detectores empregados foram os que seguem:

- Contador proporcional de ${ }^{3}$ He com número de série: 50-He3-760/38E, da empresa Centronic, com sensibilidade a nêutrons térmicos de $186 \mathrm{cps} / \mathrm{nv}$ (ver figura 4.1.-1). Este detector experimental foi posicionado a nordeste, de acordo com a figura 4-2.

- Contador proporcional de $\mathbf{B F}_{3}$ com número de série: BF3-107EB/70/50HS, da empresa Centronic, com sensibilidade a nêutrons térmicos de $150 \mathrm{cps} / \mathrm{nv}$ (ver figura 4.1.-1). Este detector experimental foi posicionado a sudeste, de acordo com a figura 4-2.

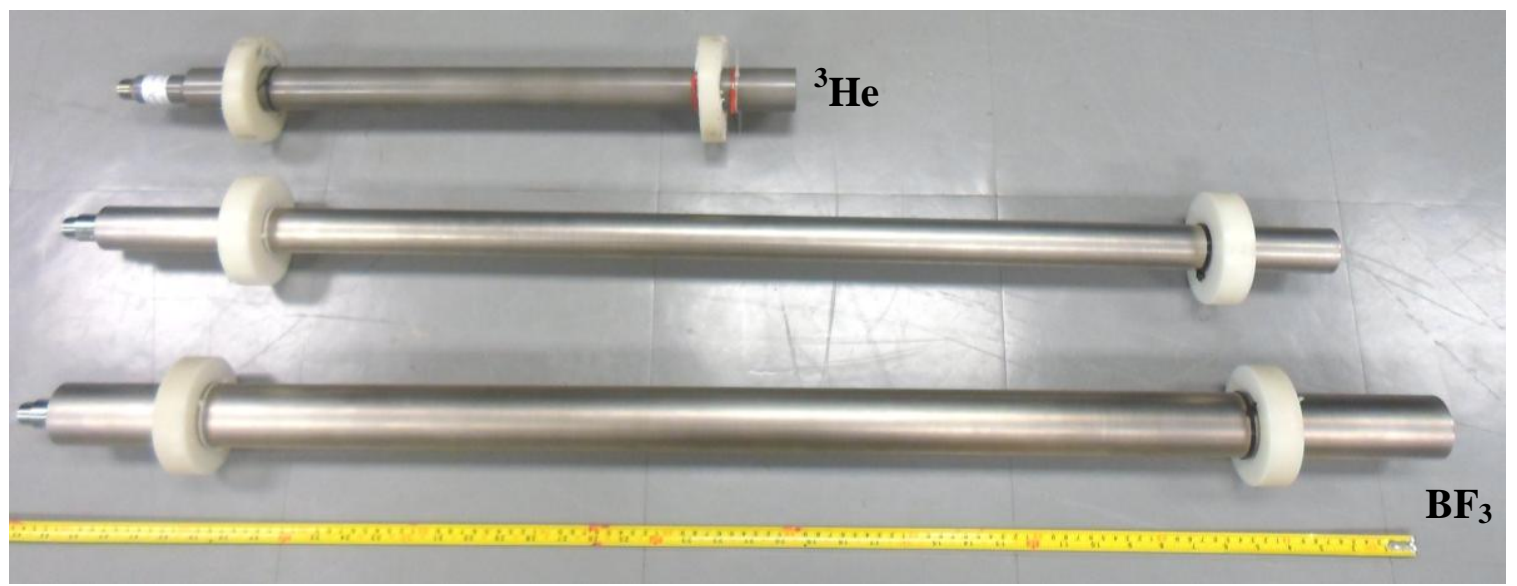

Figura4.1.-1: Fotografia dos detectores 50-He3-760/38E (o menor, mais acima na figura) e BF3-107EB/70/50HS (o maior, mais abaixo na figura). A fita métrica que aparece na fotografia mede 1,10m - Foto por Eduardo Gonnelli. 
Como pode ser observado a partir dos dados dos detectores e das figuras esquemáticas do núcleo do reator mostrados anteriormente, o detector mais sensível a nêutrons térmicos utilizado foi o contador proporcional de ${ }^{3} \mathrm{He}$. Este, por sua vez, ao ser posicionado a nordeste, pode-se dizer que ficou mais sensível ao efeito de sombreamento do banco de controle BC1. Com isso, como será visto mais adiante, quando é feito o cálculo da reatividade do sistema, os dados deste detector indicam uma reatividade mais negativa que a calculada a partir dos dados de contagens do detector proporcional de $\mathrm{BF}_{3}$, principalmente para o caso em que os bancos estão completamente inseridos.

\subsection{Parâmetros cinéticos do reator IPEN/MB-01}

Deve-se lembrar que, para um dado núcleo físsil, cada grupo "i" de nêutrons atrasados é caracterizado por dois parâmetros nucleares:

$\diamond \quad$ A proporção $\boldsymbol{\beta}_{\mathbf{i}}$ de nêutrons atrasados deste grupo entre o grupo de nêutrons emitidos por fissão (prontos e atrasados), expressa, na prática em pcm;

$\diamond \quad$ A constante de decaimento radioativo $\lambda_{\mathbf{i}}$ do precursor, isto é, da desintegração beta que vai conduzir ao núcleo capaz de emitir um nêutron.

Neste trabalho, os parâmetros neutrônicos considerados são totalmente experimentais e foram obtidos na própria instalação do reator IPEN/MB-01 ${ }^{\text {xxxiii }}$. A relação destes 
parâmetros (consideramos aqui 6 grupos de nêutrons atrasados) pode ser vista na tabela abaixo.

Tabela 4.2.-1: Parâmetros neutrônicos totalmente experimentais para o reator nuclear IPEN/MB-01.

\begin{tabular}{|c|l|l|}
\hline $\mathrm{i}$ & \multicolumn{1}{|c|}{$\beta_{\mathrm{i}}$} & \multicolumn{1}{c|}{$\lambda_{\mathrm{i}}\left(\mathrm{s}^{-1}\right)$} \\
\hline 1 & $(2,679 \pm 0,023) \mathrm{E}-4$ & $0,012456(\mathrm{fixo})$ \\
\hline 2 & $(1,463 \pm 0,069) \mathrm{E}-3$ & $0,0319 \pm 0,0032$ \\
\hline 3 & $(1,34 \pm 0,13) \mathrm{E}-3$ & $0,1085 \pm 0,0054$ \\
\hline 4 & $(3,10 \pm 0,10) \mathrm{E}-3$ & $0,3054 \pm 0,0055$ \\
\hline 5 & $(8,31 \pm 0,62) \mathrm{E}-4$ & $1,085 \pm 0,044$ \\
\hline 6 & $(4,99 \pm 0,27) \mathrm{E}-4$ & $3,14 \pm 0,11$ \\
\hline$\beta_{\text {eff }}=(7,50 \pm 0,19) \mathrm{E}-3$ & $\Lambda=31,96 \pm 1,06(\mu \mathrm{s})$ \\
\hline
\end{tabular}

\subsection{Experimento de Queda de barras (Rod Drop)}

No experimento de queda de barras o reator é aproximado do estado crítico e então é executado um procedimento de queda de barras experimental, em que os bancos de controle $\mathrm{BC} 1$ e $\mathrm{BC} 2$ caem simultaneamente, com a água ainda mantida no núcleo do reator. 
Por conta da própria sensibilidade dos detectores considerados neste trabalho, os experimentos de quedas de barras não puderam ser executados a partir do estado crítico (posição de 58,08\% de barras retiradas), tendo sido feitos a partir da posição de $57 \%$ de barras retiradas, isto é, uma posição muito próxima do estado crítico, mas, ainda assim, levemente subcrítico. Isto porque, quando no estado crítico, esses detectores saturam devido à sobreposição das cadeias de fissão. Desta forma, a posição de $57 \%$ foi a considerada por ser a mais próxima do crítico em que ainda era possível manter a integridade do espectro de energia dos detectores. Vale ressaltar que uma possível consequência deste detalhe seja um maior desvio no valor calculado para a intensidade da fonte intrínseca de nêutrons.

\subsection{Experimento de Criticalização}

No experimento de criticalização o reator parte de seu estado mais subcrítico, com os bancos de controle $\mathrm{BC} 1$ e $\mathrm{BC} 2$ totalmente inseridos, sendo retirados simultaneamente, de pouco a pouco, até que o conjunto se aproxime do estado crítico.

O propósito deste experimento é que o sistema sofra apenas pequenas perturbações, em contraste com o experimento de queda de barras. A reatividade generalizada poderá, desta maneira, ser computada entre cada estado, após a perturbação. 


\subsection{Simulações em MCNP-5}

O código MCNP, desenvolvido e mantido pelo Laboratório Nacional de Los Alamos, EUA (Los Alamos National Laboratory), é o código internacionalmente reconhecido para análise de transporte de nêutrons e raios gama pelo método de Monte Carlo ("MC" vem de "Monte Carlo" e "NP" vem de "neutral particles", ou "partículas neutras", em inglês).

No caso específico do transporte de radiação, o método de Monte Carlo consiste em seguir cada partícula desde a fonte, onde é gerada, até o momento em que termina sua história, isto é, até o momento em que a partícula escapa do sistema ou é absorvida, por exemplo. Nesse procedimento, as distribuições de probabilidade são aleatoriamente amostradas utilizando-se dados de transporte. ${ }^{\text {xxiv }}$

Este código lida com transporte de nêutrons, raios gama e transporte acoplado, isto é, transporte de raios gama secundários resultantes de interações de nêutrons. Além disso, pode também tratar de transporte de elétrons, tanto de elétrons primários quanto de elétrons secundários provenientes de interações de raios gama.

Assim, utilizando o MCNP-5 é possível simular o sistema e o experimento de forma extremamente precisa. Com o código pode-se descrever toda a geometria do núcleo do reator nuclear, levando-se em conta todos os seus componentes e materiais. É possível, desta maneira, calcular diversas grandezas de interesse à física de reatores nucleares, que 
são estimadas a partir de uma grande amostragem de histórias individuais de partículas, cujas trajetórias são simuladas. Nestes cálculos, foi utilizada a biblioteca de dados nucleares ENDF/B-VII.7.

Para cada posição de bancos de controle considerada nos experimentos desta Tese foi realizada uma simulação computacional utilizando-se o código MCNP-5. Com isto, obtém-se o que seria considerado o valor correspondente ao cálculo teórico da reatividade, para então ser feito o confronto com os resultados experimentais. 


\section{Capítulo 5}

\section{Resultados}

\subsection{Reatímetro - Teste de Consistência}

Com a finalidade de validar os cálculos executados pelo reatímetro, uma série de testes de consistência foi efetuada.

Com relação ao cálculo da reatividade clássica, um problema numérico do livro de referência de Teoria de Reatores Nucleares (LAMARSH, página 427) foi escolhido. O problema refere-se a uma inserção de $100 \mathrm{pcm}$ de reatividade em um reator térmico infinito

com ${ }^{235} \mathrm{U}$ como combustível e $\mathrm{H}_{2} \mathrm{O}$ como moderador. Neste problema, o fluxo relativo pode ser dado pela equação: 


$$
\begin{aligned}
& \phi_{T}(t)=\phi_{T 0}\left[1,44 e^{0,0182 t}-0,0359 e^{-0,013 t}-0,140 e^{-0,0598 t}\right. \\
& \left.-0,0637 e^{-0,183 t}-0,0205 e^{-1,005 t}-0,00767 e^{-2,875 t}-0,179 e^{-55,6 t}\right]
\end{aligned}
$$

A partir da equação anterior é possível obter dados de fluxo relativo para uma série temporal. Considerando um intervalo de tempo $\Delta \mathrm{t}$ de 0,2 segundos, os dados foram gerados em uma tabela, como segue:

Tabela 5.1.-1: Tabela contendo os valores para o fluxo relativo e para cada termo da

\begin{tabular}{|c|c|c|c|c|c|c|c|c|}
\hline $\mathrm{t}$ & $1^{\circ}$ termo & $2^{\circ}$ termo & $3^{\circ}$ termo & $4^{\circ}$ termo & $5^{\circ}$ termo & $6^{\circ}$ termo & $7^{\circ}$ termo & Fluxo Relativo \\
\hline 0 & 1,44 & $-0,0359$ & $-0,14$ & $-0,0637$ & $-0,0205$ & $-0,00767$ & $-0,179$ & 0,99323 \\
\hline 0,2 & 1,445251151 & $-0,035802485$ & $-0,138335573$ & $-0,061410729$ & $-0,016767205$ & $-0,004315946$ & $-2,65154 \mathrm{E}-06$ & 1,18862 \\
\hline 0,4 & 1,450521452 & $-0,035705234$ & $-0,136690934$ & $-0,059203731$ & $-0,013714105$ & $-0,002428604$ & $-3,92775 \mathrm{E}-11$ & 1,20278 \\
\hline 0,6 & 1,455810971 & $-0,035608248$ & $-0,135065848$ & $-0,057076048$ & $-0,011216937$ & $-0,001366587$ & $-5,81821 E-16$ & 1,21548 \\
\hline 0,8 & 1,461119779 & $-0,035511525$ & $-0,133460082$ & $-0,055024831$ & $-0,009174472$ & $-0,000768985$ & $-8,61856 \mathrm{E}-21$ & 1,22718 \\
\hline 1 & 1,466447946 & $-0,035415065$ & $-0,131873407$ & $-0,053047332$ & $-0,007503915$ & $-0,000432712$ & $-1,27667 \mathrm{E}-25$ & 1,23818 \\
\hline 1,2 & 1,471795544 & $-0,035318867$ & $-0,130305595$ & $-0,0511409$ & $-0,006137545$ & $-0,000243489$ & $-1,89115 \mathrm{E}-30$ & 1,24865 \\
\hline 1,4 & 1,477162641 & $-0,03522293$ & $-0,128756423$ & $-0,049302982$ & $-0,005019975$ & $-0,000137012$ & $-2,80137 \mathrm{E}-35$ & 1,25872 \\
\hline 1,6 & 1,482549311 & $-0,035127254$ & $-0,127225668$ & $-0,047531116$ & $-0,0041059$ & $-7,70976 \mathrm{E}-05$ & $-4,14969 \mathrm{E}-40$ & 1,26848 \\
\hline 1,8 & 1,487955624 & $-0,035031838$ & $-0,125713112$ & $-0,045822927$ & $-0,003358266$ & $-4,33832 \mathrm{E}-05$ & $-6,14697 \mathrm{E}-45$ & 1,27799 \\
\hline 2 & 1,493381652 & $-0,034936681$ & $-0,124218539$ & $-0,044176128$ & $-0,002746768$ & $-2,44119 \mathrm{E}-05$ & $-9,10556 \mathrm{E}-50$ & 1,28728 \\
\hline 2,2 & 1,498827467 & $-0,034841782$ & $-0,122741734$ & $-0,042588513$ & $-0,002246616$ & $-1,37367 \mathrm{E}-05$ & $-1,34881 E-54$ & 1,29640 \\
\hline 2,4 & 1,50429314 & $-0,034747141$ & $-0,121282486$ & $-0,041057953$ & $-0,001837535$ & $-7,72971 \mathrm{E}-06$ & $-1,99801 E-59$ & 1,30536 \\
\hline 2,6 & 1,509778745 & $-0,034652757$ & $-0,119840588$ & $-0,0395824$ & $-0,001502943$ & $-4,34955 \mathrm{E}-06$ & $-2,95967 \mathrm{E}-64$ & 1,31420 \\
\hline 2,8 & 1,515284354 & $-0,03455863$ & $-0,118415831$ & $-0,038159875$ & $-0,001229275$ & $-2,44751 \mathrm{E}-06$ & $-4,38418 \mathrm{E}-69$ & 1,32292 \\
\hline 3 & 1,520810039 & $-0,034464758$ & $-0,117008013$ & $-0,036788473$ & $-0,00100544$ & $-1,37723 \mathrm{E}-06$ & $-6,49432 \mathrm{E}-74$ & 1,33154 \\
\hline 3,2 & 1,526355875 & $-0,034371141$ & $-0,115616933$ & $-0,035466357$ & $-0,000822362$ & $-7,74972 \mathrm{E}-07$ & $-9,62008 \mathrm{E}-79$ & 1,34008 \\
\hline 3,4 & 1,531921935 & $-0,034277779$ & $-0,11424239$ & $-0,034191756$ & $-0,00067262$ & $-4,36081 \mathrm{E}-07$ & $-1,42503 \mathrm{E}-83$ & 1,34854 \\
\hline 3,6 & 1,537508292 & $-0,03418467$ & $-0,11288419$ & $-0,032962962$ & $-0,000550144$ & $-2,45385 \mathrm{E}-07$ & $-2,11091 \mathrm{E}-88$ & 1,35693 \\
\hline 3,8 & 1,54311502 & $-0,034091814$ & $-0,111542136$ & $-0,031778328$ & $-0,00044997$ & $-1,38079 \mathrm{E}-07$ & $-3,12691 \mathrm{E}-93$ & 1,36525 \\
\hline 4 & 1,548742194 & $-0,03399921$ & $-0,110216038$ & $-0,030636269$ & $-0,000368036$ & $-7,76978 \mathrm{E}-08$ & $-4,63191 \mathrm{E}-98$ & 1,37352 \\
\hline 4,2 & 1,554389888 & $-0,033906858$ & $-0,108905706$ & $-0,029535253$ & $-0,000301021$ & $-4,37209 \mathrm{E}-08$ & $-6,8613 \mathrm{E}-103$ & 1,38174 \\
\hline 4,4 & 1,560058177 & $-0,033814757$ & $-0,107610952$ & $-0,028473806$ & $-0,000246209$ & $-2,4602 \mathrm{E}-08$ & $-1,0164 \mathrm{E}-107$ & 1,38991 \\
\hline 4,6 & 1,565747136 & $-0,033722906$ & $-0,10633159$ & $-0,027450505$ & $-0,000201377$ & $-1,38437 \mathrm{E}-08$ & $-1,5056 \mathrm{E}-112$ & 1,39804 \\
\hline 4,8 & 1,571456841 & $-0,033631304$ & $-0,105067439$ & $-0,02646398$ & $-0,000164709$ & $-7,78989 \mathrm{E}-09$ & $-2,2302 \mathrm{E}-117$ & 1,40613 \\
\hline 5 & 1,577187368 & $-0,033539951$ & $-0,103818317$ & $-0,025512909$ & $-0,000134718$ & $-4,38341 \mathrm{E}-09$ & $-3,3036 \mathrm{E}-122$ & 1,41418 \\
\hline
\end{tabular}
equação 5.1.-1 considerando um intervalo de tempo $\Delta \mathrm{t}$ de 0,2 segundos.

A partir dos dados da primeira e da última colunas, pode-se obter o gráfico do Fluxo Relativo versus Tempo (s), que, como se pode notar, corresponde precisamente ao exemplo dado no livro de referência. 
Gráfico gerado a partir da eq. 12-35 pág. 427 Lamarsh

Fluxo Relativo x Tempo (s)

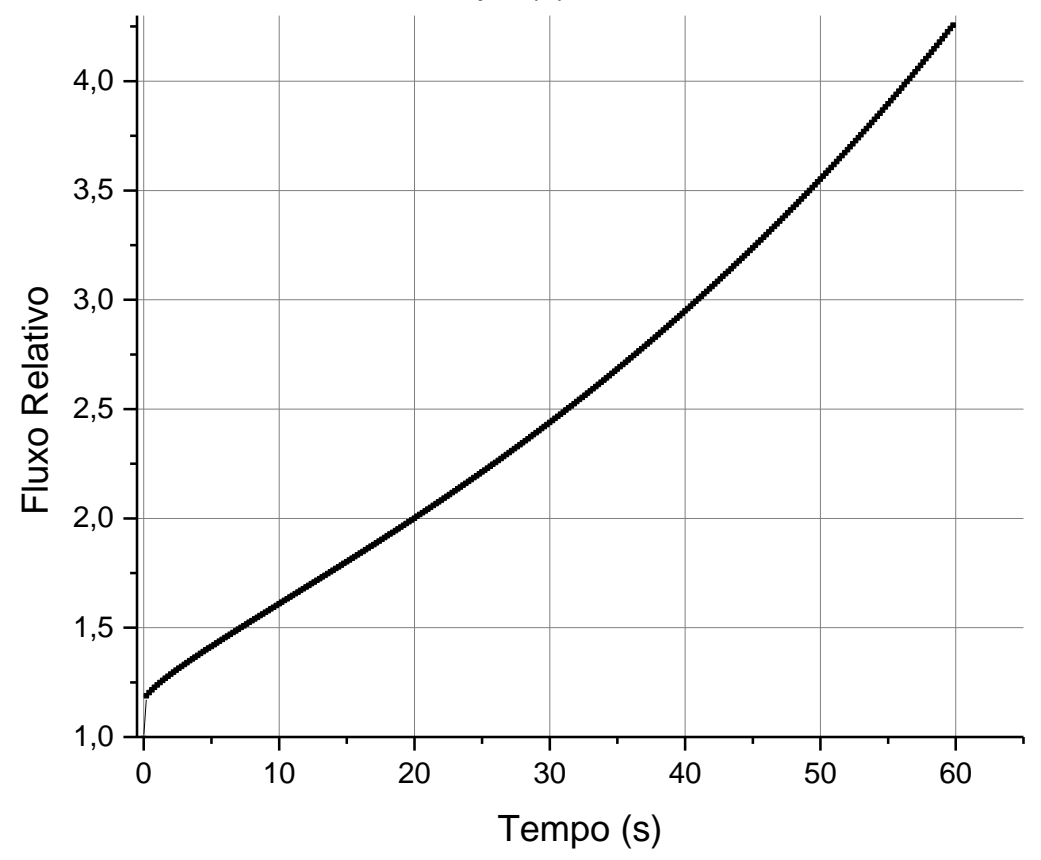

Figura 5.1.-1: Gráfico do Fluxo Relativo versus o Tempo(s).

Agora, utilizando-se os parâmetros cinéticos do ${ }^{235} \mathrm{U}$ (LAMARSH, página 100) e os dados de fluxo relativo gerados a partir da tabela 5.1.-1 como dados de entrada para o reatímetro, pode-se analisar a reatividade calculada pelo mesmo.

Tabela 5.1.-2: Parâmetros cinéticos do ${ }^{235} \mathrm{U}$ :

\begin{tabular}{|c|c|c|}
\hline & $\lambda i(1 / \mathrm{s})$ & $\beta i$ \\
\hline 1 & 0,0124 & 0,000215 \\
\hline 2 & 0,0305 & 0,001424 \\
\hline 3 & 0,111 & 0,001274 \\
\hline 4 & 0,301 & 0,002568 \\
\hline 5 & 1,14 & 0,000748 \\
\hline 6 & 3,01 & 0,000273 \\
\hline & $\Lambda=0,0001 \mathrm{~s}$ & $\beta=0,0065$ \\
\hline
\end{tabular}


Exatamente como o previsto, utilizando os dados previamente mencionados no reatímetro desenvolvido nesta Tese, foi obtida uma inserção de 100 pcm de reatividade, como pode ser visto no gráfico abaixo (figura 5.1.-2):

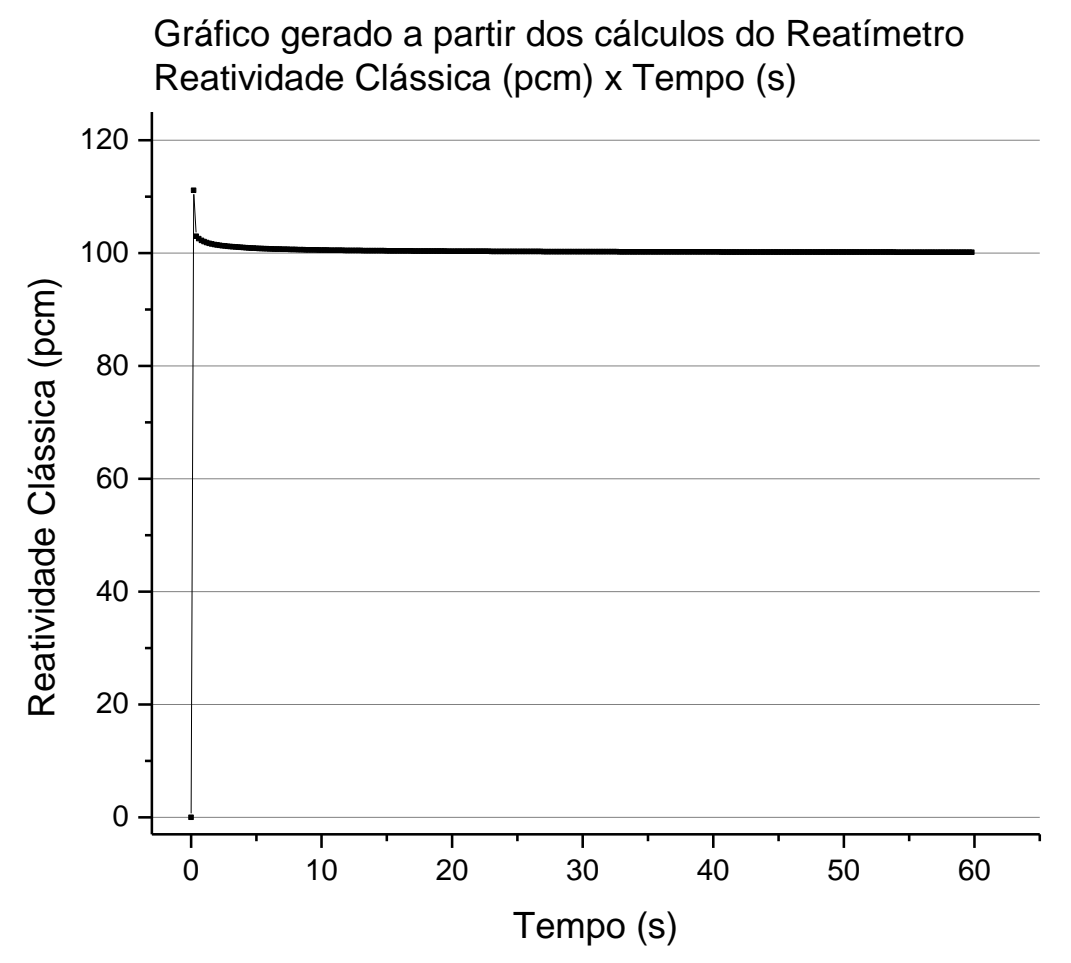

Figura 5.1.-2: Gráfico da Reatividade Clássica (pcm) versus o Tempo(s).

Com isto, considerando a concordância entre os resultados obtidos pelo reatímetro desenvolvido e os resultados de referência, demonstra-se que o cálculo da reatividade clássica está sendo executado corretamente. 
Deve-se frisar que os próximos resultados a serem apresentados foram obtidos a partir dos cálculos executados pelo reatímetro.

\subsection{Reatímetro Subcrítico}

Como visto anteriormente, o Reatímetro Subcrítico foi programado utilizando-se a linguagem LabVIEW ${ }^{\mathrm{TM}}$ e é capaz de calcular a reatividade subcrítica de acordo com a cinética pontual de Gandini. Para tanto, utiliza como dado de entrada um arquivo em .txt contendo séries temporais de contagens de nêutrons. Como dado de saída, gera um arquivo em .txt com 5 colunas contendo informações: do índice de subcriticalidade $\zeta$, dados de entrada (contagens), a concentração dos precursores de nêutrons atrasados $\mathrm{C}(\mathrm{t})$, o parâmetro Q(t) (para o cálculo da fonte intrínseca de nêutrons) e, finalmente, a reatividade generalizada $\rho_{\text {gen }}$.

Além dos dados de entrada, também são necessárias outras informações, como os parâmetros cinéticos considerados e o intervalo de tempo considerado entre as contagens do arquivo de entrada (neste trabalho foi considerado $\Delta \mathrm{t}=1 \mathrm{~s}$ ).

Pode-se acompanhar os cálculos, enquanto o programa os realiza, por meio de dois gráficos que são mostrados em seu painel frontal. Um dos gráficos nos mostra o comportamento do índice de subcriticalidade e o outro nos mostra o comportamento da reatividade generalizada com o passar do tempo. O painel frontal do reatímetro pode ser visto na figura 5.2.-1. 


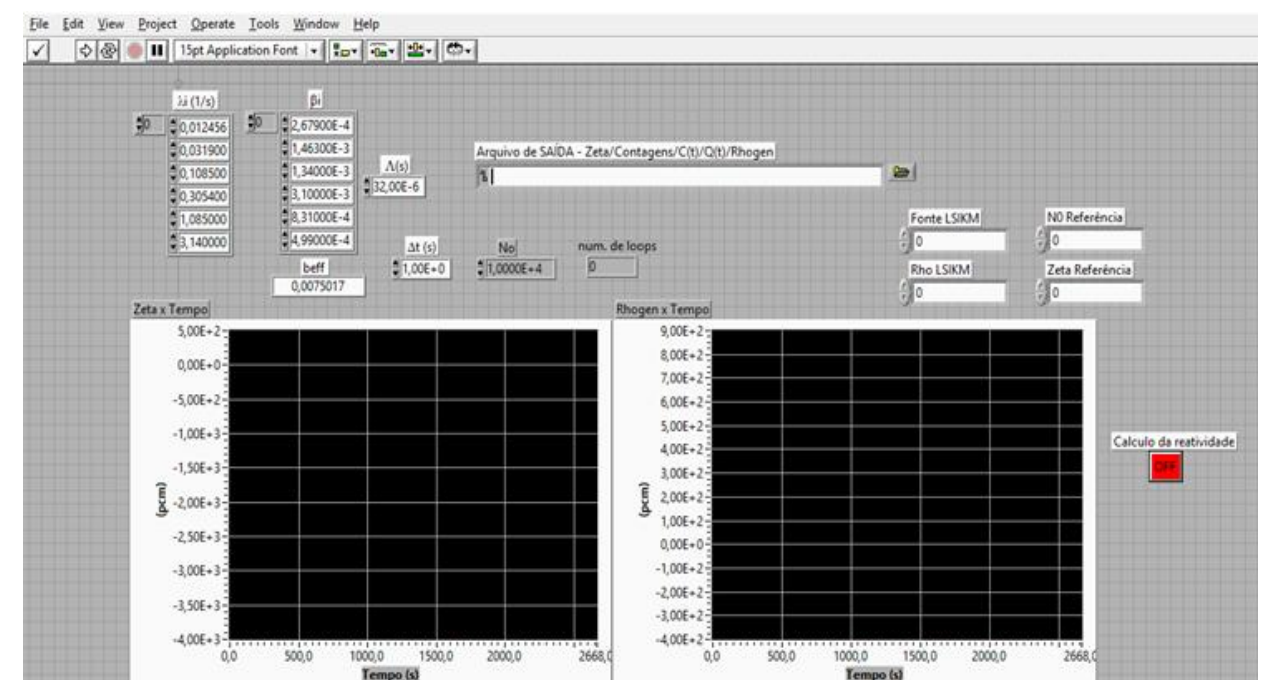

Figura5.2.-1: Painel Frontal do Reatímetro Subcrítico desenvolvido.

\subsection{Determinação da intensidade da fonte intrínseca de nêutrons}

Como detalhado no capítulo 3, sobre a Fonte Intrínseca de Nêutrons, a fonte S é expressa como a taxa efetiva de nêutrons detectados devido à fonte intrínseca. Este parâmetro, que é dependente tanto do núcleo do reator quanto do detector utilizado através da eficiência do detector $\varepsilon$, é geralmente desconhecido ${ }^{x x i x}$. Desta forma, diversos experimentos de quedas de barras (rod drop) e dois detectores foram considerados para o cálculo da intensidade da fonte.

Desta maneira, a partir de dados de experimentos de queda de barras foi possível obter os seguintes gráficos para a determinação da fonte intrínseca de nêutrons para cada detector e posição considerada: 


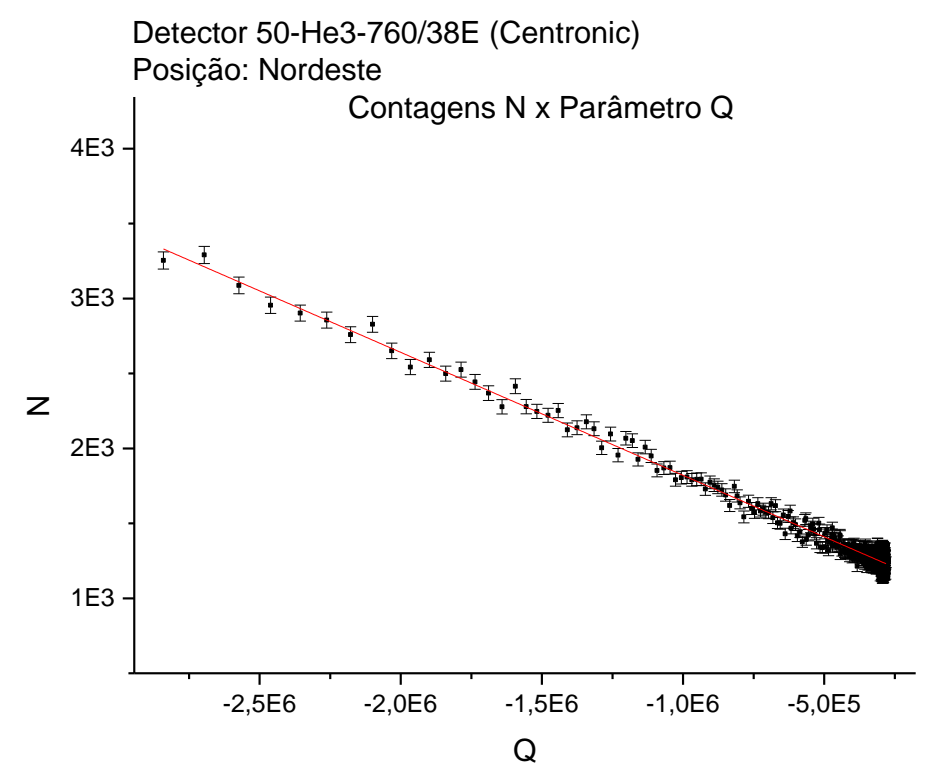

Figura 5.3.-1: Gráfico das contagens versus parâmetro Q para o detector de ${ }^{3} \mathrm{He}$.

Como se pode ver no gráfico da figura 5.3.-1, os dados das contagens $\mathrm{N}$ versus parâmetro Q distribuem-se aproximadamente ao longo de uma reta, como era o esperado para a aplicação do método LSIKM para a determinação da intensidade da fonte de nêutrons.

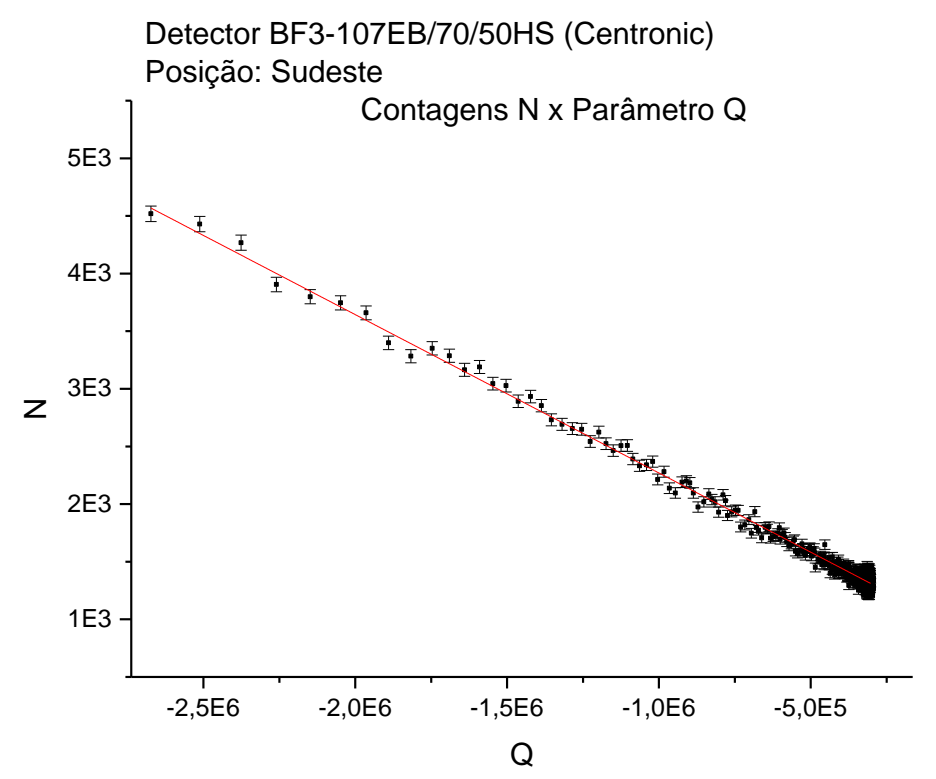

Figura 5.3.-2: Gráfico das contagens versus parâmetro $Q$ para o detector de $\mathrm{BF}_{3}$. 
Assim, como se pode ver no gráfico da figura 5.3.-2, os dados das contagens $\mathrm{N}$ versus parâmetro Q distribuem-se de maneira muito parecida com os do gráfico anterior, da figura 5.2.-1, ao longo de uma reta.

A partir do ajuste do Método dos Mínimos Quadrados em Cinética Inversa (LSIKM), como discutido no capítulo 3, foi possível obter os seguintes valores para $\rho$ (uma estimativa da reatividade clássica) e $\mathrm{S}$ (a intensidade da fonte de nêutrons intrínseca), de acordo com a equação (3.4.-7):

Tabela 5.3.-1: Estimativa da reatividade Clássica $(\rho)$ e intensidade da fonte de nêutrons intrínseca (S) obtidas pelo Método dos Mínimos Quadrados em Cinética Inversa (LSIKM) para os detectores considerados em suas determinadas posições.

\begin{tabular}{|c|c|c|}
\hline \multicolumn{2}{|c|}{ BF3 } & desvio \\
\hline$\rho(\mathbf{p c m})$ & $-3,60 \mathrm{E}+03$ & $1,29 \mathrm{E}+02$ \\
\hline S (n/s) & $1,49 \mathrm{E}+06$ & $5,38 \mathrm{E}+05$ \\
\hline
\end{tabular}

\begin{tabular}{|c|c|c|}
\hline \multicolumn{2}{|c|}{3 He } & desvio \\
\hline$\rho(p c m)$ & $-3,84 \mathrm{E}+03$ & $7,46 \mathrm{E}+01$ \\
\hline S (n/s) & $1,56 \mathrm{E}+06$ & $1,20 \mathrm{E}+05$ \\
\hline
\end{tabular}


Neste caso, era de se esperar que, como os detectores são diferentes e estavam posicionados em locais diferentes, mesmo que ligeiramente (um a nordeste e outro a sudeste), estes naturalmente fariam medidas diversas para a intensidade da fonte de nêutrons. Deve-se destacar, por exemplo, que o detector de ${ }^{3} \mathrm{He}$ estava posicionado a frente do banco de controle BC1. No entanto, pelo que pôde ser observado, considerando os desvios das estimativas da intensidade da fonte de nêutrons, os resultados obtidos a partir dos dados dos dois detectores estão em concordância.

\subsection{Queda de Barras (Rod Drop)}

Para a elaboração desta Tese foram feitos diversos experimentos de quedas de barras. A seguir, o comportamento das contagens em função do tempo para um experimento de queda de barras pode ser visto nas figuras 5.4.-1 e 5.4.-2. Como o esperado, antes e após a queda das barras as contagens apresentam um comportamento constante. 


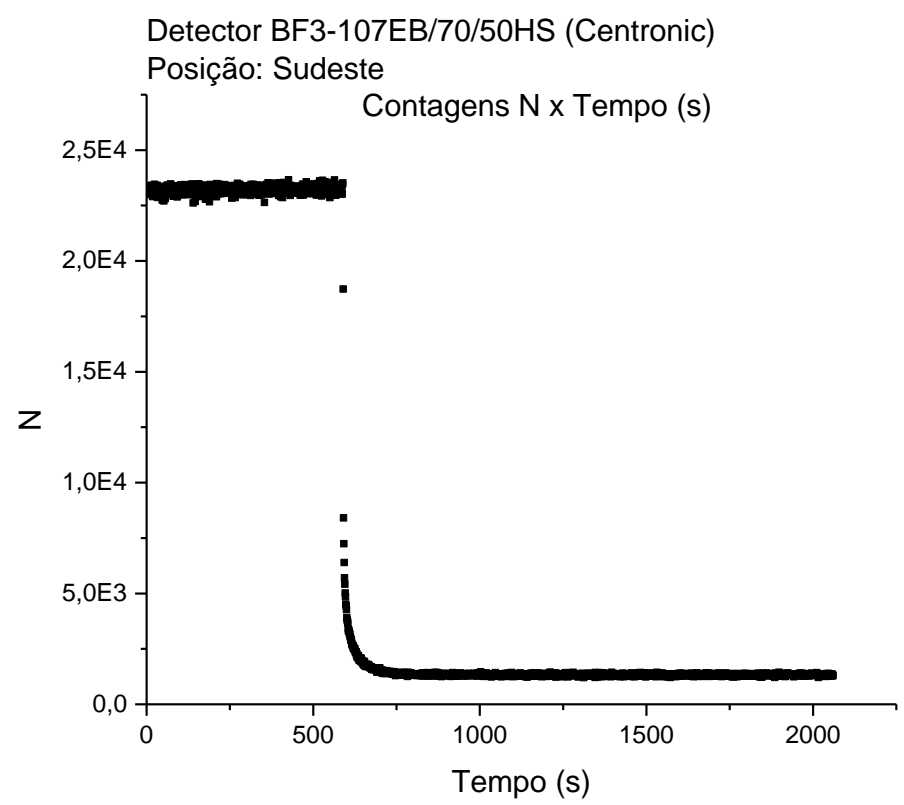

Figura 5.4.-1: Gráfico das Contagens $\mathrm{x}$ Tempo (s) para o detector de $\mathrm{BF}_{3}$ para experimento de queda de barras.

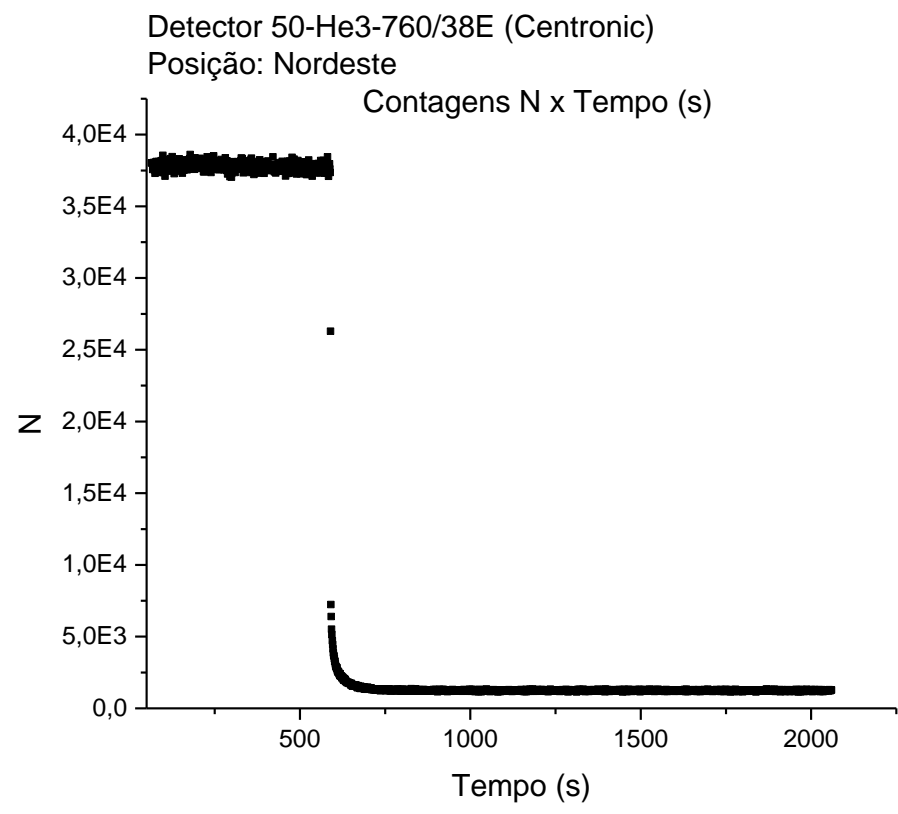

Figura 5.4.-2: Gráfico das Contagens x Tempo (s) para o detector de ${ }^{3} \mathrm{He}$ para experimento de queda de barras. 
Uma maneira de verificar se a fonte de nêutrons calculada através do ajuste realmente corresponde à fonte de nêutrons intrínseca do reator é calcular a reatividade a partir das equações de cinética pontual clássicas para o experimento de queda de barras considerando o determinado detector e a respectiva fonte obtida a partir dos cálculos de ajuste. Caso a fonte tenha sido calculada de maneira incorreta e apresente valor menor, a reatividade irá aumentar, tendendo a zero; caso a fonte tenha sido calculada de maneira incorreta e apresente valor maior, a reatividade irá diminuir, tendendo a menos infinito; em contrapartida, caso o valor calculado para a fonte esteja dentro de determinada margem, a reatividade calculada apresentará um valor estável após a queda de barras.

Desta maneira, considerando os valores obtidos para a fonte de nêutrons, pôde-se calcular via reatímetro o índice de criticalidade $\zeta$ (equivalente à reatividade clássica, neste trabalho) a partir dos dados de experimentos de queda de barras (ver figuras 5.4.-3 e 5.4.-4). 


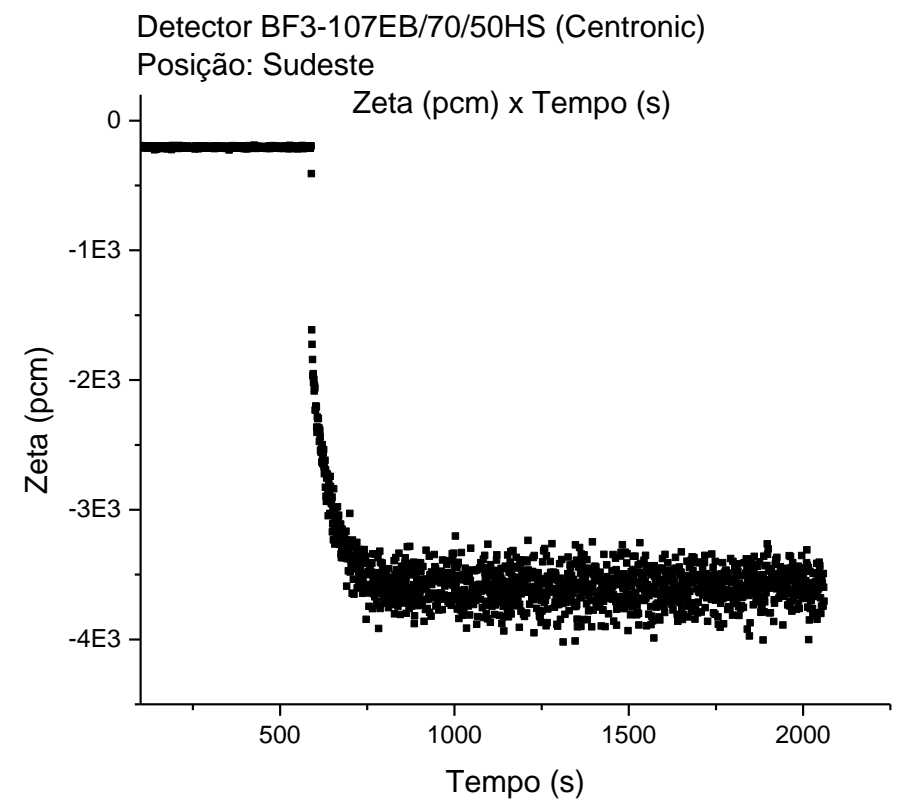

Figura 5.4.-3: Gráfico do índice de subcriticalidade $\zeta(\mathrm{pcm}) \times$ Tempo (s) para o detector de $\mathrm{BF}_{3}$ para experimento de queda de barras.

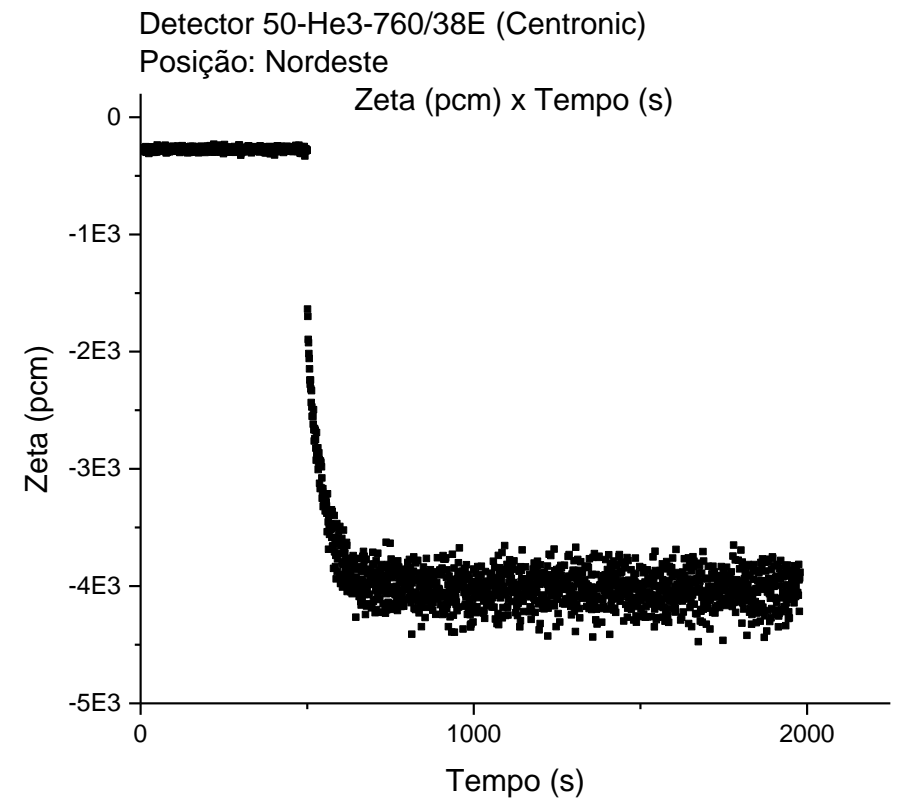

Figura 5.4.-4: Gráfico do índice de subcriticalidade $\zeta(\mathrm{pcm}) \times$ Tempo (s) para o detector de ${ }^{3}$ He para experimento de queda de barras. 
Como pode ser observado nas figuras 5.4.-3 e 5.4.-4, o índice de subcriticalidade $\zeta$, correspondente à reatividade clássica apresenta comportamento estável, em domínio subcrítico, após a queda de barras - o que indica uma estimativa correta da fonte intrínseca de nêutrons.

\subsection{Determinação da reatividade generalizada}

A partir dos dados obtidos anteriormente, foi possível, finalmente, calcular a reatividade subcrítica de acordo com a cinética pontual de Gandini.

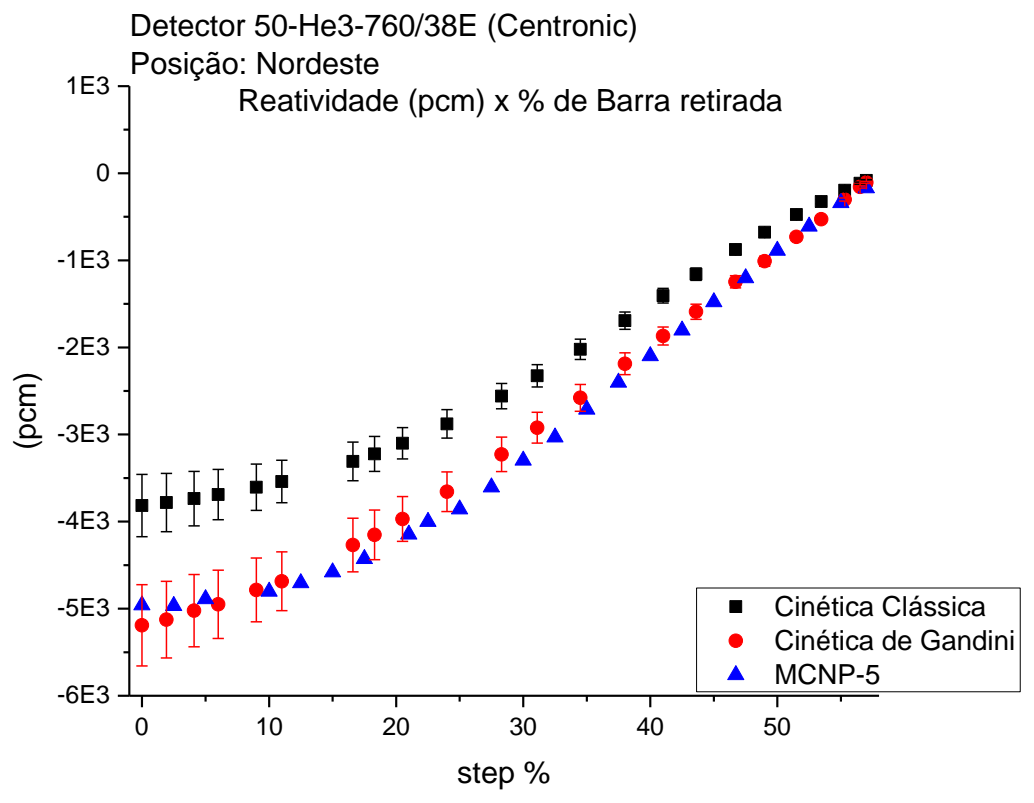

Figura 5.5.-1: Gráfico da reatividade em pcm calculada a partir da Cinética Clássica (quadrado), Cinética de Gandini (círculo) e pelo código computacional MCNP-5 (triângulo) versus a porcentagem de barra retirada, para os dados do detector de ${ }^{3} \mathrm{He}$. 


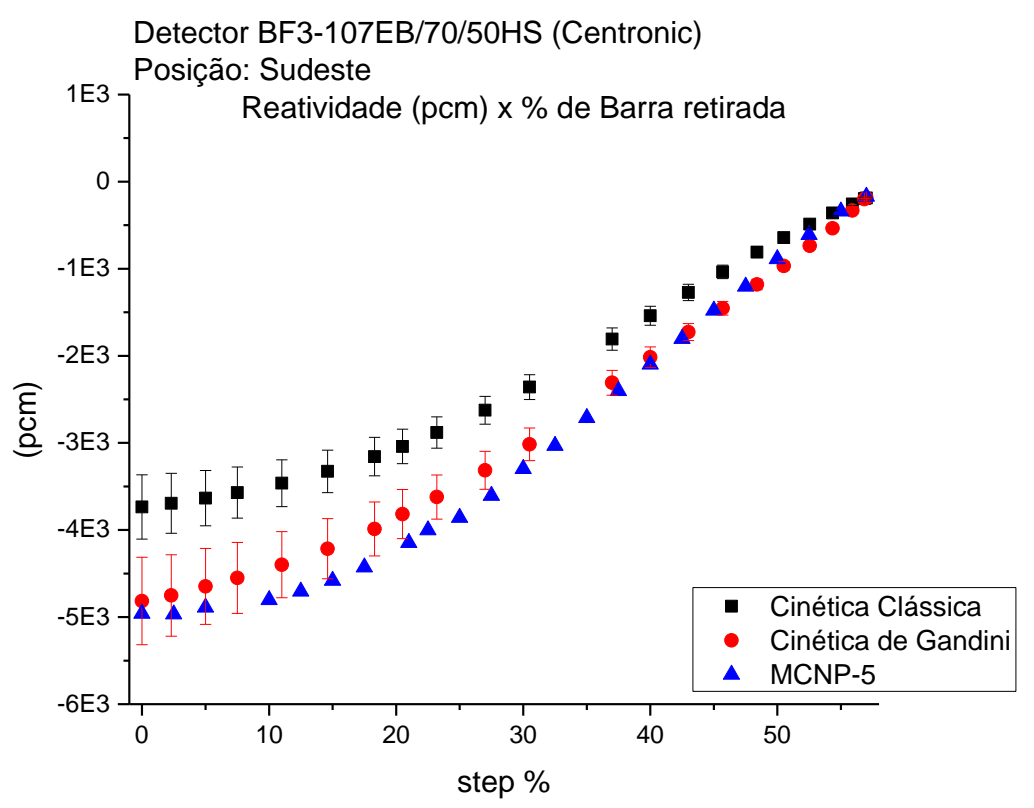

Figura 5.5.-2: Gráfico da reatividade em pcm calculada a partir da Cinética Clássica (quadrado), Cinética de Gandini (círculo) e pelo código computacional MCNP-5 (triângulo) versus a porcentagem de barra retirada, para os dados do detector de $\mathrm{BF}_{3}$.

Analisando as figuras 5.5.-1 e 5.5.-2, é possível notar que todas as curvas apresentam um formato sigmoide, como era o esperado. Adicionalmente, pode-se notar que os resultados dos três métodos concordam para a região próxima do estado crítico e apresentam cada vez mais divergências quanto mais se aproximam dos estados mais subcríticos. Neste contexto, percebe-se que a reatividade calculada a partir das equações de Cinética Clássica apresenta valores mais positivos, enquanto que a reatividade calculada a partir das equações de Gandini apresenta valores mais negativos e mais próximos do calculado pelo MCNP-5. Este comportamento já era previsto na literatura ${ }^{\text {xiii }}$. Pode-se 
notar que quanto maior o nível de subcriticalidade maior os desvios considerados, por conta de uma maior flutuação estatística nas contagens medidas pelos detectores.

\subsection{Análise de Incertezas}

Neste trabalho, As incertezas relacionadas a $\zeta$ e a $\rho_{\text {gen }}$ foram calculadas através da propagação de erros padrão. Deve-se destacar que não é considerada aqui nesta análise a correlação entre os dados. Desta maneira, a análise de incertezas do método da cinética inversa pode ser feita a partir da seguinte equação:

$$
\begin{aligned}
\sigma_{\rho}^{2}=\sigma_{\zeta}^{2}=\left(\frac{\partial \rho}{\partial \Lambda}\right)^{2} \cdot\left(\sigma_{\Lambda}\right)^{2}+\sum_{i=1}^{N F}\left(\frac{\partial \rho}{\partial \beta_{i}}\right)^{2} \cdot\left(\sigma_{\beta_{i}}\right)^{2} & +\sum_{i=1}^{N F}\left(\frac{\partial \rho}{\partial \lambda_{i}}\right)^{2} \cdot\left(\sigma_{\lambda_{i}}\right)^{2}+ \\
& +\left(\frac{\partial \rho}{\partial N}\right)^{2} \cdot\left(\sigma_{N}\right)^{2}+\left(\frac{\partial \rho}{\partial S}\right)^{2} \cdot\left(\sigma_{S}\right)^{2}
\end{aligned}
$$

Onde:

$$
\begin{aligned}
& \frac{\partial \rho}{\partial \Lambda}=\frac{1}{N(t)} \cdot \frac{d N(t)}{d t}-\frac{S}{N(t)} \\
& \frac{\partial \rho}{\partial \beta_{i}}=1-\frac{\lambda_{i} \cdot e^{\lambda_{i} \cdot t}}{N(t)} \cdot \int_{0}^{t} N\left(t^{\prime}\right) \cdot e^{\lambda_{i} \cdot t^{\prime}} \cdot d t^{\prime}-\frac{N(0)}{N(t)} \cdot e^{-\lambda_{i} \cdot t}
\end{aligned}
$$

$\frac{\partial \rho}{\partial \lambda_{i}}=\frac{1}{N(t)}\left[-\beta_{i} \cdot e^{-\lambda_{i} \cdot t} \cdot \int_{0}^{t} N\left(t^{\prime}\right) \cdot e^{\lambda_{i} \cdot t^{\prime}} \cdot d t^{\prime}+\beta_{i} \cdot \lambda_{i} \cdot e^{-\lambda_{i} \cdot t} \cdot \int_{0}^{t} N\left(t^{\prime}\right) \cdot\left(t-t^{\prime}\right) e^{\lambda_{i} \cdot t^{\prime}} \cdot d t^{\prime}\right]$

$\frac{\partial \rho(t)}{\partial N(t)}=-\frac{\rho(t)}{N(t)}$

$\frac{\partial \rho}{\partial S}=\frac{-\Lambda}{N(t)}$ 
A análise de incertezas do método da cinética de Gandini pode ser feita pela seguinte equação:

$$
\begin{aligned}
\sigma_{\rho_{g e n}}^{2}=\left(\frac{\partial \rho_{g e n}}{\partial \zeta}\right)^{2} \cdot\left(\sigma_{\zeta}\right)^{2}+\left(\frac{\partial \rho_{g e n}}{\partial \ell_{e f f}}\right)^{2} & \cdot\left(\sigma_{\ell_{e f f}}\right)^{2}+\sum_{i=1}^{N F}\left(\frac{\partial \rho_{g e n}}{\partial \beta_{i}}\right)^{2} \cdot\left(\sigma_{\beta_{i}}\right)^{2}+ \\
& +\sum_{i=1}^{N F}\left(\frac{\partial \rho_{g e n}}{\partial \lambda_{i}}\right)^{2} \cdot\left(\sigma_{\lambda_{i}}\right)^{2}+\left(\frac{\partial \rho_{g e n}}{\partial P_{N}}\right)^{2} \cdot\left(\sigma_{P_{N}}\right)^{2}
\end{aligned}
$$

Onde:

$\frac{\partial \rho_{g e n}}{\partial \varsigma}=\frac{1}{P_{N}(t)}-1$

$\frac{\partial \rho_{\text {gen }}}{\partial \ell_{\text {eff }}}=-\frac{1}{P_{N}(t)} \frac{d P_{N}(t)}{d t}$

$\frac{\partial \rho_{g e n}(t)}{\partial P_{N}(t)}=-\frac{\rho_{g e n}(t)}{P_{N}(t)}$

$\frac{\partial \rho_{g e n}}{\partial \beta_{i}}=\alpha \cdot\left[1+\frac{\lambda_{i} \cdot e^{\lambda_{i} \cdot t}}{P_{N}(t)} \cdot \int_{0}^{t} P_{N}\left(t^{\prime}\right) \cdot e^{\lambda_{i} \cdot t^{\prime}} \cdot d t^{\prime}+\frac{P_{N}(0)}{P_{N}(t)} \cdot e^{-\lambda_{i} \cdot t}\right]$

$\frac{\partial \rho_{g e n}}{\partial \lambda_{i}}=\frac{\alpha}{P_{N(t)}}\left[-\beta_{i} \cdot e^{-\lambda_{i} \cdot t} \cdot \int_{0}^{t} P_{N}\left(t^{\prime}\right) \cdot e^{\lambda_{i} \cdot t^{\prime}} \cdot d t^{\prime}+\beta_{i} \cdot \lambda_{i} \cdot e^{-\lambda_{i} \cdot t} \cdot \int_{0}^{t} P_{N}\left(t^{\prime}\right) \cdot\left(t-t^{\prime}\right) e^{\lambda_{i} \cdot t^{\prime}} \cdot d t^{\prime}\right](5.6 .-12)$ 


\section{Capítulo 6}

\section{Discussão e Conclusão}

Considerando-se os objetivos desta Tese de doutorado, é possível afirmar que foram todos atingidos. Um reatímetro subcrítico foi desenvolvido para a execução da tese, tendo sido programado em linguagem LabView ${ }^{\mathrm{TM}}$. Este programa de computador, a partir de dados de entrada provenientes de experimentos, é capaz de calcular o parâmetro Q para o cálculo da intensidade da fonte intrínseca S; o índice de subcriticalidade $\zeta$ e a reatividade generalizada $\rho_{\text {gen }}$ de acordo com a teoria de Cinética Pontual para Sistemas Subcríticos de Gandini. Diversos testes de consistência foram realizados, mostrando que o programa é capaz de realizar todos os cálculos corretamente. A intensidade da fonte intrínseca de nêutrons foi estimada para dois detectores de nêutrons $\left({ }^{3} \mathrm{He}\right.$ e $\left.\mathrm{BF}_{3}\right)$ em duas posições especificadas considerando-se o arranjo do núcleo padrão. Pelo que pôde ser observado, considerando os desvios das estimativas da intensidade da fonte de nêutrons, os resultados obtidos a partir dos dados dos dois detectores estão em concordância. 
Com relação à reatividade, considerando as figuras 5.5.-1 e 5.5.-2, quando comparamos os resultados obtidos a partir das equações de Cinética Clássica (neste trabalho identificados com o parâmetro $\zeta$ da Teoria de Gandini) e a partir das equações de Gandini com os valores obtidos pela simulação computacional em MCNP-5, vemos que as curvas apresentam um formato sigmoide, como era o esperado. Além disso, pode-se notar que os resultados dos três métodos concordam para a região próxima do estado crítico e apresentam cada vez mais divergências quanto mais se aproximam dos estados mais subcríticos. Neste contexto, percebe-se que a reatividade calculada a partir das equações de Cinética Clássica apresenta valores mais positivos, enquanto que a reatividade calculada a partir das equações de Gandini apresenta valores mais negativos e mais próximos do calculado pelo MCNP-5. Este comportamento já era previsto ${ }^{\text {xiii }}$. Pode-se notar que quanto maior o nível de subcriticalidade maior os desvios considerados, por conta de uma maior flutuação estatística nas contagens medidas pelos detectores.

Finalmente, considerando-se os resultados apresentados, pode-se concluir que a estimativa da reatividade subcrítica a partir dos dados experimentais utilizando-se o método de Gandini se aproxima muito dos valores calculados pelo código MCNP-5. 


\section{Referências Bibliográficas}

i SALVATORES, M. et. Al., "MUSE-1: A first experiment at MASURCA to validate the physics of Subcritical Multiplying Systems relevant to ADS", 2nd ADTT Conf., Kalmar, Sweden, June 1996.

ii REUSS,P., "Neutron Physics", EDP Sciences, 2008.

iii RUBBIA, C. et. Al., "The TRADE Experiment: Status of the Project and Physics of the spallation Target", PHYSOR-2004, The Physics of Fuel Cycles and Advanced Nuclear Systems: Global Developments, Chicago, Illinois, April 25-29, 2004.

iv YONG-QIAN, S.; ZHU, Q.-F.; TAO, H. "Review and research of the neutron source multiplication method in nuclear critical safety". Nucl. Technology, v. 149, n.1, p.122, 2004.

v PINTO, L. N.; GONNELLI, E. ; DOS SANTOS, A. "Control rod calibration and reactivity effects at the IPEN/MB-01 reactor". XXXVI BRAZILIAN WORKSHOP ON NUCLEAR PHYSICS, 2014, São Sebastião. v. 1625. p. 140-145.

vi VENDRYES, G. Génie Atomique. Primeiro Tomo, Capítulo: L'état sous-critique et l'approche des conditions critiques. Ed. Presses Universitaires de France, Paris, 1960. 
vii TSUJI, M., Advanced Measurement Methods in a Zero-Power Reactor Physics Test of PWRs. Core Management of Light Water Reactor, AESJ-KNS Summer School, Kyushu University, Fukuoka, Japan, August 6-8, 2008.

viii SUZUKI, E., TSUNODA, T. "A Reactivity Meter and Its Application”, Journal of Nuclear Science and Technology, 1:6, 210-218, 1964.

ix MIJUIN, D. "L'accident de Criticité de Tokaï Mura", Clefs CEA nº 45, Automne 2001.

x TSUJI, M., SUZUKI, N., SHIMAZU,Y. "Subcriticality Measurement by Neutron Source Multiplication Method with a Fundamental Mode Extraction", Journal of NUCLEAR SCIENCE and TECHNOLOGY, Vol. 40, No. 3, p. 158-169, March 2003.

xi SHIMAZU,Y., TSUJI, M. “Applicability of Point Reactor Kinetic Equations for Subcriticality Monitoring”, Proceedings of Korean Nuclear Society Spring Meeting Cheji, Korea, May 2001.

xii FURET, J., "Sécurité dans le contrôle des piles atomiques", Comissariat à l'Énergie Atomique, Rapport CEA No. 2174, 1962.

xiii AMBLARD, J-C., "La mesure de la reactivité dans un reacteur nucléaire", Comissariat à l'Énergie Atomique, Rapport CEA No. 3375, 1968. 
xiv SUZUKI, E.; TSUNODA, T., "A reactivity meter and its application", Journal of Nuclear Science and Technology, v. 1, No. 6, p. 210-218, 1964.

XV SILVA, C., "Uma nova função importância para a análise de sistemas subcríticos", Tese (Doutorado) - Universidade Federal do Rio de Janeiro, COPPE, Rio de Janeiro, 2011.

xvi ANTOLIN, M. Q., "Simulação de um reator subcrítico tipo ADS via Monte Carlo para validação de cálculo da reatividade", Tese (Doutorado) - Universidade Federal do Rio de Janeiro, COPPE, Rio de Janeiro, 2013.

xvii DULLA, S.; RAVETTO, P., "Interpretation of local flux measurements in subcritical systems and reactivity determination", Hindawi Publishing Corporation, Science and Technology of Nuclear Installations, Article ID 629039, 13 pages, 2012.

xviii NISHIHARA, K.; IWASAKI, T.; UDAGAUA, Y., "A new static and dynamic one-point equation and analytic and numerical calculations for subcritical system", Journal of Nuclear Science and Technology, v. 481, p. 481-492, 2003.

xix BAJGL, J.; KRÝS, V.; ŠVARNÝ, J., "An analysis of reactivity prediction during the reactor start-up process", Kerntechnik, v. 80, No. 4, p. 339-348, 2015. 
xx KURAMOTO, R. Y. R.; MIRANDA, A. F., "Subcritical Reactivity Measurements at ANGRA 1 Nuclear Power Plant", International Nuclear Atlantic Conference - INAC 2011, Belo Horizonte, Brasil, Outubro de 2011.

xxi Rolls-Royce Control \& Monitoring Systems - Reactivity Meter. < http://www.rolls-royce.com/customers/nuclear/instrumentation-and-control/control-and-

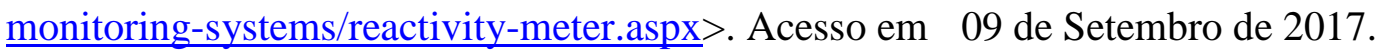

xxii NOVAK, P. et al., "Development of fast pulse processing algorithm for nuclear detectors and its utilization in LabVIEW-based Mössbauer spectrometer", Journal of Instrumentation, v. 9, 2014.

xxiii GANDINI, A.; SALVATORES, M., "The Physics of Subcritical Multiplying Systems", Journal of Nuclear Science and Technology, v. 39, No. 6, p. 673 686, 2002.

xxiv DOS SANTOS, A.; LEE, S. M.; DINIZ, R.; JEREZ, R., “A New Experimental Approach for Subcritical Reactivity Determination of Multiplying Systems", Annals of Nuclear Energy, v. 59, p. 243-254, 2013.

xxv BELL, G. I.; GLASSTONE, S., "Nuclear Reactor Theory”, Van Nostrand Reinhold Company, New York, 1970.

xxvi REUSS,P., “Neutron Physics”, EDP Sciences, 2008. 
xxvii RAMOS, A. W. Controle Estatístico de Processos para Processos Contínuos e em Bateladas. Ed. Edgard Blucher, São Paulo, 2000.

xxviii TAMURA, S., "Signal Fluctuation and Neutron Source in Inverse Kinetics Method for Reactivity Measurement in the Sub-critical Domain”, Journal of Nuclear Science and Technology, v. 40, No. 3, p. 153-157, 2003.

xxix SHIMAZU,Y.; TSUJI, M., “Applicability of Point Reactor Kinetic Equations for Subcriticality Monitoring”, Proceedings of Korean Nuclear Society Spring Meeting, Cheji, Korea, Maio de 2001.

xxx PERRET, G., JAMMES, C., IMEL, G., DESTOUCHES, C., CHAUSSONNET, P., LAURENS, J.M., SOULE, R., THOMAS, G. M., ASSAL, W., FOUGERAS, P., BLAISE, P., HUDELOT, J-P., PHILIBERT, H., BIGNAN, G. "Determination of Reactivity by a Revised Rod-Drop Technique in the MUSE-4 Programme - Comparison with Dynamic Measurements". Seventh Information Exchange Meeting on Actinide and Fission Product Partitioning and Transmutation, Jeju, Korea, 2002.

xxxi TAMURA, S. "Signal Fluctuation and Neutron Source in Inverse Kinetics Method for Reactivity Measurement in the Sub-critical Domain”, Journal of Nuclear Science and Technology, 40[3], 153-157, 2003. 
xxxii TANINAKA, H., HASHIMOTO, K., PYEON, C. H., SANO, T., MISAWA, T., UNESAKI, H., SUGIYAMA, W., OHSAWA, T. "Determination of Subcritical Reactivity of a Thermal Accelerated- Driven System from Beam Trip and Restart Experiment". Journal of Nuclear Science and Technology, 48[6], 873-879, 2012.

xxxiii DINIZ, R. Obtenção das Constantes de Decaimento e Abundâncias Relativas de Nêutrons Atrasados Através da Análise de Ruído em Reatores de Potência Zero. São Paulo, Tese (Doutorado), Instituto de Pesquisas Energéticas e Nucleares, 2005.

xxxiv ZAMBONI, C. B., YORIAZ, H., et alli. Fundamentos da Física de Nêutrons. Capítulo 8: O Método de Monte Carlo para Transporte de Nêutrons, Editora Livraria da Física, São Paulo, 2007. 